\title{
ON THE TOPOLOGY OF PARAMETRIC OPTIMAL CONTROL
}

\author{
GERHARD-W. WEBER ${ }^{1}$
}

(Received 5 May 1995; revised 28 November 1995)

\begin{abstract}
We will study one-parameter families $\left(\mathscr{P}_{s}^{\prime \prime}\right)_{s \in[\alpha . \beta]}$ of differentiable optimal control problems given by:

$$
\mathscr{P}^{\prime \prime}(s) \quad\left\{\begin{array}{l}
\operatorname{Min} \mathscr{I}^{\prime \prime}(s, x):=\int_{a}^{h} L(s, t, x(t), u(s, t)) d t, \quad \text { where } \\
\dot{x}=F(s, t, x, u) \quad(t \in[a, b]) \\
H(s, t, x, u)=0 \quad\left(t \in\left\{t_{0}^{\prime}, \ldots, t_{0}^{u \prime}\right\}\right) \\
G(s, t, x, u, y) \geq 0,\left(y \in Y^{\prime}(s, t, x, u), t \in[a, b]\right) \\
H_{a}^{h}(s, a, x(a), u(s, a), b, x(b), u(s, b))=0 \\
G_{a}^{h}(s, a, x(a), u(s, a), b, x(b), u(s, b), y) \geq 0 \\
\quad\left(y \in Y^{2}(s, a, x(a), u(s, a), b, x(b), u(s, b))\right) .
\end{array}\right.
$$

Here, at given times $t$ the inequality constraint functions are of semi-infinite nature, the objective functional may also be of max-type. For each $s \in \mathbb{R}$ the problem $\mathscr{P}_{s}^{\prime \prime}$ is equivalent to a one-parameter family $\left(\underline{P}_{s}(t)\right)_{t \in[a, b]}$ of differentiable optimization problems. From these the consideration of generalized critical trajectories, such as a local minimum trajectory, comes into our investigation. According to a concept introduced by Hettich, Jongen and Stein in optimization, we distinguish eight types of generalized critical trajectories. Under suitable continuity, compactness and integrability assumptions, those problems, which exclusively have generalized critical points being of one of these eight types, are generic. We study normal forms and characteristic examples, locally around these trajectories.

Moreover, we indicate the related concept of structural stability of optimal control problems $\mathscr{g}^{\prime \prime}$ due to the topological behaviour of the lower level sets under small data perturbations. Finally, we discuss the numerical consequences of our investigations for pathfollowing techniques with jumps.
\end{abstract}

\section{Introduction, model and motivation}

During the eighties, Jongen, Jonker and Twilt were able to introduce and to work out a Morse theory for nonlinear differentiable optimization ( $c f$. [27]). They made use of

\footnotetext{
'Department of Mathematics, Darmstadt University of Technology, Darmstadt, Germany.

(C) Australian Mathematical Society, 1998, Serial-fee code 0334-2700/98
} 
methods from differential topology. In particular, their presentation and investigation of five "generic" types of generalized critical points, for example, Kuhn-Tucker points, turned out to be a markstone for the qualitative understanding of nonlinear problems $\underline{P}_{\mathscr{F}}$ of minimization under finitely many equality or inequality constraints ( $c f$. [29, 30]). To be more precise, the property of genericity originally refers to the set of all $(f, \underline{H}, \underline{G})$ defining those one parametric optimization problems whose generalized critical points only belong to one of these five types. Genericity which we introduce in Section 2, is a stronger condition than density (cf. also [62]).

The numerical evaluation which takes account of this generic classification was elaborated by Guerra Vasquez [19], and others. For the qualitative and numerical evaluation it is geometrically easier and very helpful to investigate the problems $\underline{P}_{\mathscr{F}}(s)$, locally around $(s, \underline{x})$, in normal form, where $\underline{x}$ is a generalized critical point.

After a corresponding contribution of Rupp [57] in the meantime, recently Hettich, Jongen and Stein extended that classification from finite to semi-infinite optimization admitting now infinitely many inequality constraints. They arrived at eight generic types ( $c f .[20])$.

We remark and later on remember the fact that these generic classifications can moreover be extended to the nondifferentiable case with the objective function being of maximum-type $f(s, \underline{x})=\max _{k \in K} v_{k}(s, \underline{x})$ [39]. Besides the typical argumentation with perturbations the main idea consists in an interpretation of this nondifferentiable problem in $\mathbb{R}^{n}$ as a differentiable problem in $\mathbb{R}^{n+1}$. This idea is worked out in $[64,65]$.

Now, we take these investigations over into the infinite-dimensional context of optimal control theory. We study the behaviour of a problem in such a variational setting locally around, for example, a local minimum trajectory $x=\bar{x}^{\prime \prime}$ and maybe close to a locally minimal control variable $u=\bar{u}$. However, we are interested in the larger entity of generalized critical trajectories (embedding). These are motivated and induced by the intimate relation between optimal control and optimization that is founded in the flow coming from the controlling differential equation. Taking account of this relation and a careful topological reasoning, the author extended Guddat's and Jongen's topological concept of structural stability of an optimization problem [18,37, 64], namely of its lower level sets with respect to small data perturbations, into optimal control theory ( $c f .[66])$. Moreover, in the case of compactness of the feasible set, it turned out that this condition can again essentially be characterized by means of both a Mangasarian-Fromovitz type condition ( $c f$. [49], see also [17]) on the feasible set, and a condition of strong stability (in the sense of Kojima; $c f .[43,44]$ ), on the KuhnTucker trajectories. Hereby we have for a moment ignored the aspect of optimality with respect to the control variable, which shall soon also be reflected (Remark 2). Those trajectories are the most important generalized critical trajectories. We can again introduce eight generic types of generalized critical trajectories and study the behaviour of the optimal control problem around each of these trajectories. For 
this purpose we locally represent the problem by an easy one which is called its normal form. Such a normal form might be achieved by a canonical change of local coordinates and it exhibits the characteristics of the problem at a generalized critical trajectory. Hence, it supports both a better geometrical understanding and the numerical efforts in treating the control problem.

We remark that in the past it had been conjectured, for example, by Malanowski [47], that there is a relation between semi-infinite optimization and optimal control theory with respect to the problems' structures and qualities. This paper is a small contribution on this behalf and on to a better understanding in the qualitative theory of optimal control [45].

Let $C^{k}(\mathscr{U}, \mathbb{R})$ for any open set $\mathscr{U} \subseteq \mathbb{R}^{n}$ denote the space of real valued, $k$-times continuously differentiable functions over $\mathscr{U}$. By $C^{k}(\mathscr{M}, \mathbb{R})$, where $\mathscr{M}=[a, b]$, or $=[\alpha, \beta] \times[a, b](\alpha<\beta ; a<b)$, we denote the set of all functions $w: \mathscr{M} \rightarrow \mathbb{R}$ which can be extended to a function $\tilde{w} \in C^{k}(\mathscr{U}, \mathbb{R})$ ( $\mathscr{U}$ being some open neighbourhood of $\mathscr{M}$ ) that gives us the derivatives of $w$ at the boundary. Here we fix the index sets $I:=\{1, \ldots, m\}$ and $I_{a}^{b}:=\left\{1, \ldots, m_{a}^{b}\right\}$.

The final and underlying desire consists in tracing the given maybe hard problem, $\mathscr{P}_{\mathscr{S} \mathscr{S}}^{\prime \prime}(\beta)$, back to a problem, $\mathscr{P}_{\mathscr{S} \mathscr{\mathscr { S }}}^{\prime \prime}(\alpha)$, which is connected with it and more easy to resolve (continuation, homotopy method). Then numerical pathfollowing techniques might work in order approximatively to find a solution of the original problem $\mathscr{P}_{\mathscr{S} \mathscr{S}}^{\prime \prime}(\beta)$. Hence we look at the following family of problems $\left(\mathscr{P}_{s}^{\prime \prime}=\right) \mathscr{P}_{\mathscr{S} \mathscr{\mathscr { A }}}^{\prime \prime}(s)(s \in[\alpha, \beta], \alpha<\beta)$ :

$$
\mathscr{P}_{\mathscr{S} \mathscr{S}}^{u}(s)\left\{\begin{array}{l}
\operatorname{Min}_{x} \mathscr{I}^{u}(s, x):=\int_{a}^{b} L(s, t, x(t), u(s, t)) d t, \quad \text { where } \\
\dot{x}=F(s, t, x, u) \quad(t \in[a, b]) \\
H(s, t, x, u)=0 \quad\left(t \in\left\{t_{0}^{\prime}, \ldots, t_{0}^{w}\right\}\right) \\
G(s, t, x, u, y) \geq 0 \quad\left(y \in Y^{\prime}(s, t, x, u), t \in[a, b]\right) \\
H_{a}^{h}(s, a, x(a), u(s, a), b, x(b), u(s, b))=0 \\
G_{a}^{h}(s, a, x(a), u(s, a), b, x(b), u(s, b), y) \geq 0 \\
\quad\left(\underline{y} \in Y^{2}(s, a, x(a), u(s, a), b, x(b), u(s, b))\right) .
\end{array}\right.
$$

Here, the notation is condensed and, in particular, the $s$ - and $t$-dependence of $u$ or $x$ is not written out. Due to the index sets $I, J, l_{a}^{h}, J_{a}^{h}$, we refer to functions $f_{k} \in C^{4}\left(\mathbb{R}^{n+q+2}, \mathbb{R}\right)(k \in\{1, \ldots, n\}), u_{\ell} \in C^{4}\left(\mathbb{R}^{2}, \mathbb{R}\right)(\ell \in\{1, \ldots, q\})$, maybe restricted on the rectangle $[\alpha, \beta] \times[a, b], h_{i} \in C^{3}\left(\mathbb{R}^{n+4+2}, \mathbb{R}\right)(k \in I),\left(h_{o}^{b}\right)_{i} \in$ $C^{3}\left(\mathbb{R}^{2(n+q)+3}, \mathbb{R}\right)\left(i \in I_{a}^{b}\right), G \in C^{3}\left(\mathbb{R}^{n+p+q+2}, \mathbb{R}\right), G_{a}^{b} \in C^{3}\left(\mathbb{R}^{2(n+q)+p+3}, \mathbb{R}\right)$ and $L \in$ $C^{3}\left(\mathbb{R}^{n+q+2}, \mathbb{R}\right)$. We use the notation $F, u, H, H_{a}^{b}$ in order to comprise these coordinate functions as vectors, respectively. The functions $G, G_{a}^{h}$ could also be vector valued. Moreover, the state constraints of equality type are demanded at given discrete times $t_{0}^{k} \in[a, b](k \in\{1, \ldots, w\})$ with $t_{0}^{k}<t_{0}^{k+1}(k \in\{1, \ldots, w-1\})$. 
Because of the underlying infinite dimension of the state variables and of the control variables as well, problems of the form above are of infinite nature. Looking at their structure and their stability behaviour these problems and related ones were investigated in [66]. These infinite problems do also possess a semi-infinite character coming from the maybe infinite number of inequality constraints either at each time $t \in[a, b]$ or being a boundary value condition. We might call the problems $\mathscr{P}_{\mathscr{S} \mathscr{I}}^{u}(s)$ semi-infinite optimal control problems, symbolically: $\mathscr{S} \mathscr{I}$. Whenever both setvalued mappings $Y^{j}(\cdot)(j \in\{1,2\})$ are pointwise of finite cardinality, then we call our optimal control problems finite. This special case is symbolically indicated by $\mathscr{F}$ and $\mathscr{P}_{\mathscr{F}}^{u}(s)$.

For $Y^{j}(\cdot)(j \in\{1,2\})$ we demand upper semi-continuity [33, 64], and compactness of all values in $\mathbb{R}^{p}$ whose two sets of unions of all of them, respectively, are also supposed to be disjoint. According to [20], in the parametric case we call this condition Assumption 1.

Let us in particular think that pointwise these index sets are feasible sets in the sense of finite optimization, hence they are of the form

$$
\begin{array}{r}
Y^{1}(s, t, x, u)=\left\{\underline{y} \in \mathbb{R}^{p} \mid \hat{h}_{k}^{1}(s, t, x, u, \underline{y})=0\left(k \in A^{\prime}\right),\right. \\
\left.\hat{g}_{\ell}^{1}(s, t, x, u, \underline{y}) \geq 0\left(\ell \in B^{1}\right)\right\}, \\
Y^{2}(s, a, x(a), u(s, a), b, x(b), u(s, b)) \\
=\left\{\underline{y} \in \mathbb{R}^{p} \mid \hat{h}_{k}^{2}(s, a, x(a), u(s, a), b, x(b), u(s, b), \underline{y})=0\left(k \in A^{2}\right),\right. \\
\left.\hat{g}_{\ell}^{2}(s, a, x(a), u(s, a), b, x(b), u(s, b), \underline{y}) \geq 0\left(\ell \in B^{2}\right)\right\},
\end{array}
$$

where $A^{j}, B^{j}$ are analogous to $I, J$, respectively, with cardinalities $\left|A^{j}\right|,\left|B^{j}\right|<\infty$. We comprise all the defining functions which define the constraints on $\underline{y}$, due to both $j=1$ and $j=2$ and due to the different sets where these functions are defined on. Namely, therefore we introduce the notation $\widehat{H}(\cdot), \widehat{G}(\cdot)$. For these vectors of functions, which shall also be of class $C^{3}$, the part on upper semi-continuity in Assumption 1 holds generically (see $[33,64]$ ). We consider them together with the other functions which define the optimal control problem, and now we precisely call the problem for the parameter $s \in[a, b]$ :

$$
\begin{gathered}
\mathscr{P}_{\mathscr{S} \mathscr{S}}^{u \prime}(s)=\mathscr{P}_{\mathscr{S} \mathscr{S}}^{u(s \cdot)}\left(L(s, \cdot), F(s, \cdot),(H(s, \cdot), G(s, \cdot)),\left(H_{a}^{b}(s, \cdot), G_{a}^{b}(s, \cdot)\right),\right. \\
(\widehat{H}(s, \cdot), \widehat{G}(s, \cdot))) .
\end{gathered}
$$

REMARK 1. The problem description presented above can be generalized in several directions. Namely, the index sets $Y^{1}, Y^{2}$, pointwise defined, may consist of vectors having different length $p^{1}, p^{2}$, respectively. Each of these sets may also be replaced by a finite number of sets. Moreover, the discrete times $t_{0}^{1}, \ldots, t_{0}^{w}$ may in a $C^{3}$-manner 
depend on the parameter $s$. We call the $w$-tuple, consisting of these (component) functions $t_{0}^{\nu}(s)(v \in\{1, \ldots, w\})$ the function $\underline{C}(s)$, say over $\mathbb{R}$. Moreover the time interval $[a, b]$ may be substituted by means of a of union of finitely many disjoint intervals $\biguplus_{\mu=1}^{v}\left[a_{\mu}(s), b_{\mu}(s)\right](s \in[\alpha, \beta])$ with ordered endpoint functions $a_{\mu}, b_{\mu}$ of class $C^{3}$, say over $\mathbb{R}: a \leq a_{\mu-1}(s)<b_{\mu-1}(s)<a_{\mu}(s) \leq b(\mu \in\{2, \ldots, v\})$, comprised by $\underline{A}$ and $\underline{B}$, the list of functions describing the optimal control problem would become extended such that we get $\mathscr{P}_{\mathscr{S} \mathscr{S}}^{u(s .)}\left(L(s, \cdot), F(s, \cdot),(H(s, \cdot), G(s, \cdot)),\left(H_{a}^{b}(s, \cdot), G_{a}^{b}(s, \cdot)\right)\right.$, $(\widehat{H}(s, \cdot), \widehat{G}(s, \cdot)),(\underline{A}(s), \underline{B}(s), \underline{C}(s)))$. Moreover, we may replace $\mathscr{I}^{\prime \prime}(s, x)$ by an objective functional being of maximum-type, that is, by $\max _{\underline{\underline{a}} \in Z(s)} \mathscr{I}^{u}(s, x, \underline{z})$. Then the index set $Z(s)$ of functionals in competition would, in analogy with $Y^{1}(s, a, \underline{x}, \underline{u})$, $Y^{2}\left(s, a, \underline{x}^{1}, \underline{u}^{1}, b, \underline{x}^{2}, \underline{u}^{2}\right)$, be supposed to fulfill the following condition.

ASSUMPTION 1'. $Z$ is upper semi-continuous and compact-valued.

Here $Z$ would also be supposed to have the form $Z(s)=\left\{\underline{z} \in \mathbb{R}^{r} \mid \bar{h}_{k}(s, z)=\right.$ $\left.0(k \in C), \bar{g}_{\ell}(s, z) \geq 0(\ell \in D)\right\}$ with finite index sets $C, D$ and defining functions of class $C^{3}$. Then our entire problem would be called $\mathscr{P}_{\mathscr{S} \mathscr{S} \cdot \max }^{\prime \prime}(s):=\mathscr{P}_{\mathscr{S} \mathscr{S}}^{u(s .)}(L(s, \cdot)$, $F(s, \cdot),(H(s, \cdot), G(s, \cdot)),\left(H_{a}^{b}(s, \cdot), G_{a}^{b}(s, \cdot)\right),(\widehat{H}(s, \cdot), \widehat{G}(s, \cdot)),(\underline{A}(s), \underline{B}(s), \underline{C}(s))$, $(\bar{H}(s, \cdot), \bar{G}(s, \cdot)))$. Finally, in [66] it is possible to let the objective functional be a sum of the integral functional with an additional finite-valued penalty-like functional $l(x(a), u(a), x(b), u(b))$, or, in this research, with the parametrized functional $l(s, x(a), u(s, a), x(b), u(s, b))$. Then $l$ and $l(s, \cdot)$, respectively, would enlarge the list of functional parameters (data) of our optimal control problem.

In order to work out the basic topological features of our optimal control problem we may concentrate on the differentiable problem $\mathscr{P P}_{\mathscr{S} \mathscr{g}}^{u \prime}(s)=\mathscr{P}_{\mathscr{S} \mathscr{S}}^{u(s .)}(L(s, \cdot), F(s, \cdot)$, $\left.(H(s, \cdot), G(s, \cdot)),\left(H_{a}^{h}(s, \cdot), G_{a}^{b}(s, \cdot)\right),(\widehat{H}(s, \cdot), \widehat{G}(s, \cdot))\right)$. Let us for the "nondifferentiable minimization" of a generic function(al) of continuous selection type (for example, max-type) only remark that one can on the one hand separately study normal forms of the differentiable objective function(al)s being involved. On the other hand one can also use the transformation of such an objective function from optimization into an easier nondifferentiable normal form, which was with the help of singularity theory presented by Jongen and Pallaschke [32].

REMARK 2. In [66] a survey on three approaches to the structure of optimal control problems is given, where the latter are easier than $\mathscr{P}_{\mathscr{S} \mathscr{S}}^{\prime \prime}(s)$. Namely, all the equality constraints are of boundary type, there are no boundary conditions of inequality type, and only finitely many of the inequality constraints for all $t \in[a, b]$. However, the minimization jointly refers to $(x, u)$. Then, under generic assumptions the problems' structure can on the one hand be expressed by finite optimization problems in $\underline{u}$ and semi-infinite problems in $\underline{x}$. With the minimizing in $\underline{u}$ which is based on the minimum principle, we have the opportunity to take account of the goal of optimality in $u$, jointly 
with $x$. On the other hand, hereby a certain further auxiliary flow structure is utilized in order to reflect the infinite dimensionality of optimal control. Those semi-infinite problems are defined by means of inserting control variables of the form $u_{v}^{0}(s, t, \cdot)$. Referring to these, the explanations of this paper hold, too. Moreover, one can also work out this paper such that $u(s, \cdot)$ becomes treated as the implicitly defined function $u_{v}^{0}(s, \cdot)$. Then it is no longer a part of the list of given functional data. However, the technicalities would rapidly rise.

Finally, we remark that our problems $\mathscr{P}_{\mathscr{S} \mathscr{S}}^{\prime \prime}(s)$ can be considered as pieces within the piecewise structure that generically appears for those easier problems from [66]. Hereby, the control variables are allowed to be piecewise differentiable.

Indeed, the first step of our investigation is to trace the optimal control problem back to an optimization problem or, in other words, to a one parameter family of equivalent optimization problems. This is possible because of the parametrized flow $\Phi^{\prime \prime}(s, \cdot)$ due to our time-dependent differential equation (a system due to the parameter $s)$ under a suitable integrability (controllability) assumption $(c f .[2,66])$.

Namely, we make the following assumption.

ASSUMPTION $2(L B)$. With continuous nonnegative(-valued) functions $\alpha, \beta$, we have that

$$
\|F(s, t, \underline{x}, \underline{u})\| \leq \alpha(s, t, \underline{u})\|\underline{x}\|+\beta(s, t, \underline{u}) \quad \text { for all }(s, t, \underline{x}, \underline{u}) \in \mathbb{R}^{n+q+2} .
$$

In our typical problems we may, for example, look at linear differential equations which always fulfill Assumption 2 and can often easier, or explicitly, be evaluated. For harder differential equations, numerical methods should approximately be used.

Because of Assumption 2 we may conclude (see, for example, [2, Theorem 7.6 and 7.8]) that all initial value problems due to the prescribed differential equation are solvable over the whole time space $\mathbb{R}$. (Problems for which Assumption 2 does nol hold, that is, where a violation of controllability raises, will be treated in a following paper.) Here we get all the parametrized $C^{4}$-solutions $x(\cdot)=x^{s}(\cdot)$, over $\mathbb{R}$ and in particular over $[a, b]$, namely $x^{s}(\cdot)=\Phi^{u}\left(s, \underline{x}_{0},-t_{0}+\cdot\right)$, where $\underline{x}_{0}=x^{s}\left(t_{0}\right)$, and a twoparameter family of $C^{4}$-diffeomorphisms $\Phi^{\prime \prime}(s, \cdot, t)((s, t) \in[\alpha, \beta] \times \mathbb{R})$. Moreove we have that

$$
\Phi^{u}(s, \underline{x}, 0)=\underline{x}, \quad \Phi^{u}\left(s, \Phi^{u}\left(s, \underline{x}, t^{1}\right), t^{2}\right)=\Phi^{u}\left(s, \underline{x}, t^{1}+t^{2}\right) \quad\left(\underline{x} \in \mathbb{R}^{n} ; t^{1}, t^{2} \in \mathbb{R}\right) .
$$


For $\underline{x}=x(t), t \in \mathbb{R}$, we define

$$
\begin{gathered}
\underline{f}^{\prime \prime}(s, t, \underline{x}):=\mathscr{I}^{\prime \prime}\left(s, \Phi^{\prime \prime}(s, \underline{x},-t+\cdot)\right) \\
\underline{h}_{i}^{\prime \prime}(s, t, \underline{x}):=\left\{\begin{array}{l}
h_{\ell}\left(s, t_{0}^{k}, \Phi^{\prime \prime}\left(s, \underline{x},-t+t_{0}^{k}\right), u\left(s, t_{0}^{k}\right)\right) \\
\text { for } i=(\ell-1) w+k, \ell \in I, k \in\{1, \ldots, w\} \\
\left(h_{a}^{b}\right)_{p}\left(s, a, \Phi^{\prime \prime}(s, \underline{x},-t+a), u(s, a), b, \Phi^{\prime \prime}(s, \underline{x},-t+b), u(s, b)\right) \\
\quad \text { or } i=m w+p, p \in I_{a}^{h}
\end{array}\right. \\
\underline{g}_{1}^{\prime \prime}(s, t, \underline{x},(\underline{y}, \xi)):=G\left(s, \xi, \Phi^{\prime \prime}(s, \underline{x},-t+\xi), u(s, \xi), \underline{y}\right) \\
:=\left(G_{a}^{b}\right)_{p}\left(s, a, \Phi^{\prime \prime}(s, \underline{x},-t+a), u(s, a), b, \Phi^{\prime \prime}(s, \underline{x},-t+b), u(s, b), \underline{y}\right)
\end{gathered}
$$

Because of the index sets for the inequality constraints depending on $\underline{x}$, we establish the same dependence as above, inserting the flows:

$$
\begin{aligned}
& \underline{\hat{h}}_{i}^{l \cdot u}(s, t, \underline{x},(\underline{y}, \xi)):=\hat{h}_{i}^{l}\left(s, \xi, \Phi^{\prime \prime}(s, \underline{x},-t+\xi), u(s, \xi), \underline{y}\right), \quad \text { for } i \in A^{\prime}, \\
& \underline{\hat{h}}_{i}^{2 . u}(s, t, \underline{x},(\underline{y}, \xi)):=\hat{h}_{i}^{2}\left(s, a, \Phi^{\prime \prime}(s, \underline{x},-t+a), u(s, a), b,\right. \\
& \left.\Phi^{\prime \prime}(s, \underline{x},-t+b), u(s, b), \underline{y}\right), \quad \text { for } i \in A^{2}, \\
& \underline{\hat{g}}_{\ell}^{\prime . u}(s, t, \underline{x},(\underline{y}, \xi)):=\hat{g}_{\ell}^{1}\left(s, \xi, \Phi^{u}(s, \underline{x},-t+\xi), u(s, \xi), \underline{y}\right), \quad \text { for } \ell \in B^{\prime} \text {, } \\
& \underline{\hat{g}}_{\ell}^{2 . u t}(s, t, \underline{x},(\underline{y}, \xi)):=\hat{g}_{\ell}^{2}\left(s, a, \Phi^{\prime \prime}(s, \underline{x},-t+a), u(s, a), b\right. \text {, } \\
& \left.\Phi^{u}(s, \underline{x},-t+b), u(s, b), \underline{y}\right), \quad \text { for } \ell \in B^{2}, \\
& \underline{\hat{h}}_{i}^{u}:= \begin{cases}\underline{\hat{h}}_{i}^{1 . u}, & \text { for } i \in A^{\prime} \\
\underline{\hat{h}}_{i-\left|A^{\prime}\right|,}^{2 . u}, & \text { for } i \in\left\{\left|A^{\prime}\right|+1, \ldots,\left|A^{\prime}\right|+\left|A^{2}\right|\right\},\end{cases} \\
& \underline{\hat{g}}_{\ell}^{u \prime}:= \begin{cases}\underline{\hat{g}}_{\ell}^{1 . u}, & \text { for } \ell \in B^{1} \\
\xi-a, & \text { for } \ell=\left|B^{1}\right|+1 \\
-\xi+b, & \text { for } \ell=\left|B^{1}\right|+2 \\
\underline{\hat{g}}_{\ell-\left|B^{\prime}\right|-2}^{2 . u}, & \text { for } \ell \in\left\{\left|B^{1}\right|+3, \ldots,\left|B^{1}\right|+\left|B^{2}\right|+2\right\} .\end{cases}
\end{aligned}
$$

Note that $\underline{g}_{2}^{u}, \underline{\hat{h}}_{i}^{2 . u}\left(i \in A^{2}\right), \underline{\hat{g}}_{\ell}^{2 . u}\left(\ell \in B^{2}\right)$ do not depend on $\xi$. Moreover, we put

$$
\begin{gathered}
\underline{I}:=\left\{1, \ldots, m w+m_{a}^{h}\right\}, \quad \underline{J}:=\left\{1, \ldots, s z+s_{a}^{b}\right\}, \\
\widehat{I}:=\left\{1, \ldots,\left|A^{\prime}\right|+\left|A^{2}\right|\right\}, \quad \underline{J}:=\left\{1, \ldots,\left|B^{1}\right|+\left|B^{2}\right|+2\right\}, \\
\underline{H}^{u}:=\left(\underline{h}_{i}^{u}\right)_{i \in \underline{I}}, \quad \underline{G}^{u}:=\left(\underline{g}_{1}^{\prime}, \underline{g}_{2}^{u}\right), \quad \underline{\widehat{H}}:=\left(\underline{\hat{h}}_{i}^{u}\right)_{i \in \underline{I}}, \quad \underline{\widehat{G}}:=\left(\underline{\hat{g}}_{i}^{u}\right)_{i \in \underline{\underline{I}}} .
\end{gathered}
$$

We state that $\underline{f}^{u}, \underline{H}^{\prime \prime}, \underline{G}^{u}, \widehat{\widehat{H}}^{\prime \prime}, \underline{\widehat{G}}^{u}$ are three times continuously differentiable. 
For each $s \in[\alpha, \beta]$ we use the notation $\underline{u}=u(s, t)$, we take account of the constraints $a \leq \xi \leq b$ (see also $\underline{\hat{g}}_{\ell}^{\prime \prime}, \ell \in\left\{\left|B^{1}\right|+1,\left|B^{2}\right|+2\right\}$ ), set

$$
\begin{aligned}
\underline{Y}^{\prime}(s, t, \underline{x}, \underline{u}):=\left\{(\underline{y}, \xi) \in \mathbb{R}^{p} \times \mathbb{R} \mid \underline{\hat{h}}_{i}^{l \cdot u}(s, t, \underline{x},(\underline{y}, \xi))\right. & =0\left(i \in A^{1}\right), \\
\underline{g}_{\ell}^{l \cdot u}(s, t, \underline{x},(\underline{y}, \xi)) & \left.\geq 0\left(\ell \in B^{1}\right), a \leq \xi \leq b\right\}
\end{aligned}
$$

and choosing, for example, $\xi=a$ :

$$
\begin{aligned}
\underline{Y}^{2}(s, t, \underline{x}, \underline{u}):=\left\{(\underline{y}, a) \in \mathbb{R}^{p} \times \mathbb{R} \mid \underline{\hat{h}}_{i}^{2 . u}(s, t, \underline{x},(\underline{y}, a))\right. & =0\left(i \in A^{2}\right), \\
\underline{\hat{g}}_{\ell}^{2 . u}(s, t, \underline{x},(\underline{y}, a)) & \left.\geq 0\left(\ell \in B^{2}\right)\right\} .
\end{aligned}
$$

Now our problem $\mathscr{P}_{\mathscr{S} \mathscr{S}}^{\prime \prime}(s)$ is equivalent with each of the following optimization problems

$$
\begin{aligned}
& \left.\underline{P}_{\mathscr{S} \mathscr{I}}^{\prime \prime}(s, t):=\underline{P}_{\mathscr{S P}}\left(\underline{f}^{\prime \prime}(s, t, \cdot), \underline{H}^{\prime \prime}(s, t, \cdot), \underline{G}^{u}(s, t, \cdot)\right),\left(\widehat{H}^{\prime \prime}(s, t, \cdot), \underline{\widehat{G}}^{\prime \prime}(s, t, \cdot)\right)\right), \\
& \text { also written } \underline{P}_{\mathscr{S} \mathscr{S}}\left(\underline{f}^{u}(s, t, \cdot), \underline{H}^{\prime \prime}(s, t, \cdot), \underline{G}^{u}(s, t, \cdot)\right) \text {, } \\
& \left\{\begin{array}{l}
\text { Minimize } \underline{f}^{u}(s, t, \cdot) \text { on } \underline{M}_{s, t}^{u}, \text { where } \\
\underline{M}_{s, t}^{\prime \prime}=\underline{M}\left[\underline{H}^{\prime \prime}(s, t, \cdot), \underline{G}^{\prime \prime}(s, t, \cdot)\right] \\
:=\left\{\underline{x} \in \mathbb{R}^{\prime \prime} \mid \underline{h}_{i}^{u}(s, t, \underline{x})=0(i \in \underline{l}), \underline{g}_{j}^{u}(s, t, \underline{x},(\underline{y}, \xi)) \geq 0\right. \\
\left.\left((\underline{y}, \xi) \in \underline{Y}^{j}(s, t, \underline{x}, \underline{u}), j \in\{1,2\}\right)\right\}
\end{array}\right.
\end{aligned}
$$

( $t \in[a, b]$ ). As these problems are indeed equivalent, it is sufficient to deal with $t=a$. In order to work within topological manifolds, we assume that the cardinality $|\underline{I}|:=m w+m_{a}^{b}$ of $\underline{I}$ is less than $n$, and, similarly, $\left|A^{\prime}\right|+\left|A^{2}\right|<p+1$.

Besides the notation of the feasible set of $\underline{P}_{\mathscr{S} \mathscr{I}}^{u}(s, t)$ given above, we write $M_{s}^{u}$ for the feasible set of state variables of the problem $\mathscr{P}_{\mathscr{S} \mathscr{S}}^{u}(s)$. Let us denote the sets of active inequality constraints of the problems $\underline{P}_{\mathscr{S} \mathscr{I}}^{u}(s, t)$ and $\mathscr{P}_{\mathscr{S} \mathscr{S}}^{u}(s)$ at a feasible point $\underline{x}$ or at a feasible trajectory $x$, respectively, in the following way:

$$
\begin{gathered}
\underline{Y}_{0}^{j}(s, t, \underline{x}, \underline{u})=\left\{(\underline{y}, \xi) \in \underline{Y}^{j}(s, t, \underline{x}, \underline{u}) \mid \underline{g}_{j}^{u}(s, t, \underline{x},(\underline{y}, \underline{\xi}))=0\right\} \quad(j \in\{1,2\}) \\
Y_{0}^{1}(s, t, x, u):=\left\{\underline{y} \in Y^{1}(s, t, x, u) \mid G(s, t, x, u, \underline{y})=0\right\} \\
\text { and } Y_{0}^{2}(s, a, x(a), u(s, a), b, x(b), u(s, b)) \text { analogously. }
\end{gathered}
$$

By $D f, D^{2} f\left(D_{x} f, D_{x}^{2} f\right)$ we denote the row-vector of first or the matrix of second derivatives of $f \in C^{\overline{2}}(\mathscr{M}, \mathbb{R}), \mathscr{M} \subseteq \mathbb{R}^{k}$, respectively (or, due to the variable $\underline{x}$ ). In the case $f \in\left(C^{1}(\mathscr{M}, \mathbb{R})\right)^{\ell}, D f$ (and $D_{x} f$ ) denote the functional matrix. Note that for $k=1$ we have $D f=\dot{f}$.

DEFINITION 1. Let a parameter value $\bar{s} \in[\alpha, \beta]$ and a control variable $u \in\left(C^{4}([\alpha, \beta]\right.$ $\times[a, b], \mathbb{R}))^{n}$ be given. 
(a) A point $\underline{\underline{x}} \in \mathbb{R}^{\prime \prime}$ is called a generalized critical point of the optimization problem $P_{\mathscr{S} \mathscr{S}}^{\prime \prime}(\bar{s}, \bar{t})$, where $\bar{t} \in[a, b]$ is a given time, if the vectors

$$
\begin{aligned}
& D_{\underline{x}} \underline{f}^{\bar{u}}(\bar{s}, \bar{t}, \underline{\bar{x}}), D_{\underline{x}} \underline{h}_{i}^{\bar{u}}(\bar{s}, \bar{t}, \underline{x}) \quad(i \in \underline{l}), \\
& D_{\underline{x}} \underline{g}_{j}^{\bar{u}}(\bar{s}, \bar{t}, \underline{\bar{x}},(\underline{y}, \underline{\xi})) \quad\left((\underline{y}, \xi) \in \underline{Y}_{0}^{j}(\bar{s}, \bar{t}, \underline{\bar{x}}, \bar{u}(\bar{s}, \bar{t})), j \in\{1,2\}\right)
\end{aligned}
$$

are linearly dependent.

(b) A trajectory $\vec{x} \in\left(C^{4}([a, b], \mathbb{B})^{n}\right.$ is called a generalized critical trajectory of the optimal control problem $\mathscr{P}_{\mathscr{S} \mathscr{S}}^{\prime \prime}(\bar{s})$ if there is a time $t \in[a, b]$ with $\underline{x}=\bar{x}(t)$ and $\bar{x}(t)$ is a generalized critical point of $\underline{P}_{\mathscr{S} \mathscr{S}}^{\prime \prime}(\bar{s}, t)$.

We introduce the generalized critical point sets and the generalized critical trajectory sets as follows:

$\underline{\Sigma}_{g c}(t):=\left\{(s, \underline{x}) \in \mathbb{B} \times \mathbb{R}^{\prime \prime} \mid \underline{x}\right.$ is a generalized critical point for $\underline{P}_{\mathscr{S} \mathscr{S}}^{\prime \prime}(s, t)$, $s \in[\alpha, \beta]\}$,

$\Sigma_{g c}:=\left\{(s, x) \in \mathbb{B} \times\left(C^{4}([a, b], \mathbb{R})\right)^{n} \mid x\right.$ is a generalized critical trajectory for

$$
\begin{gathered}
\left.\mathscr{P}_{\mathscr{S} \mathscr{I}}^{\prime \prime}(s), s \in[\alpha, \beta]\right\} \\
=\left\{(s, x) \in \mathbb{R} \times\left(C^{4}([a, b], \mathbb{R})\right)^{\prime \prime}\left\{(s, x(t)) \in \underline{\Sigma}_{g c}(t) \text { for some } t \in[a, b],\right.\right. \\
s \in[\alpha, \beta]\} .
\end{gathered}
$$

The generalized critical points and generalized critical trajectories need not to be feasible. However, feasibility is one property which we demand for the subsequent important and large class of generalized critical points and generalized critical trajectories.

DEFINITION 2. Let a parameter value $\bar{s} \in[\alpha, \beta]$ and a control variable $u \in\left(C^{4}([\alpha, \beta]\right.$ $\times[a, b], \mathbb{R}))^{4}$ be given.

(a) A point $\underline{\bar{x}} \in \underline{M}_{\bar{s}, i}^{\prime \prime}$ is called a Kuhn-Tucker point of the optimization problem $\mathscr{P}_{\mathscr{S} \mathscr{I}}^{\prime \prime}(\bar{s}, \bar{t})$ where $\bar{t} \in[a, b]$ is a given time, if there exist nonnegative integers $\kappa_{1}, \kappa_{2}$, indices $\left(\underline{y}^{j . \ell}, \xi^{j . \ell}\right) \in \underline{Y}_{0}^{j}(\bar{s}, \bar{t}, \underline{x}, \underline{\bar{u}})$ and real numbers $\lambda_{i}=\lambda_{i}^{u}(\bar{s}, \bar{t}, \bar{x})(i \in$ $\underline{I}), \mu_{j, \ell}=\mu_{j, \ell}^{u}(\overline{\bar{s}}, \bar{t}, \underline{\bar{x}})\left(\ell \in\left\{1, \ldots, \kappa_{j}\right\}, j \in\{1,2\}\right)$ satisfying the following relations $\mathrm{KT} 1_{\bar{s}}^{u}, 2_{\bar{s}}^{u}$ :

$\mathrm{KT} 1 \frac{u}{s}$.

$$
\begin{aligned}
D_{\underline{x}} \underline{f}^{\prime \prime}(\bar{s}, \bar{t}, \underline{\bar{x}})= & \sum_{i \in \underline{I}} \lambda_{i} D_{\underline{x}} \underline{h}_{i}^{\prime \prime}(\bar{s}, \bar{t}, \underline{\bar{x}}) \\
& +\sum_{j \in\{1.2\}} \sum_{\ell \in\left\{1 \ldots . . . \kappa_{j}\right\}} \mu_{j . \ell} D_{\underline{x}} \underline{g}_{j}^{\prime \prime}\left(\bar{s}, \bar{t}, \underline{\bar{x}},\left(\underline{y^{j . \ell}}, \underline{\xi}^{j . \ell}\right)\right),
\end{aligned}
$$


$\mathbf{K T} 2{ }_{\bar{s}}^{\prime \prime} . \quad \mu_{j . \ell} \geq 0\left(\ell \in\left\{1, \ldots, \kappa_{j}\right\}, j \in\{1,2\}\right)$.

The numbers $\lambda_{i}, \mu_{j, \ell}$ are called Lagrange multipliers.

(b) A trajectory $\bar{x} \in\left(C^{4}([a, b], \mathbb{R})\right)^{n}$ is called a Kuhn-Tucker trajectory of the optimal control problem $\mathscr{P}_{\mathscr{S} \mathscr{S}}^{\prime \prime}(\bar{s})$ if there is a time $t \in[a, b]$ with $\bar{x}=\bar{x}(t)$ and $\bar{x}(t)$ is a Kuhn-Tucker point of $\underline{P}_{\mathscr{S} \mathscr{S}}^{\prime \prime}(\bar{s}, t)$.

In analogy with the corresponding sets due to "generalized criticality" we introduce the Kuhn-Tucker point sets and the Kuhn-Tucker trajectory sets,

$$
\underline{\Sigma}_{K T}(t) \quad(t \in[a, b]), \quad \Sigma_{K T}
$$

respectively. We note that each Kuhn-Tucker trajectory $x$ is feasible, that is, $x \in M_{s}^{\prime \prime}$ with the corresponding $s$.

From the numerical point of view we shall discuss the underlying geometrical structure of these critical sets on which pathfollowing techniques with jumps (see $[1,19,57])$ from optimization may be applied with respect to finitely many times or equivalently, because of the equivalence of our optimization problems, to one time $t$. For more underlying theory in parametric optimization see [7] and, from the viewpoint of (structural) stability, [34].

Taking account of the flows and our definitions, the six items which are given in the sequel follow after a little thought.

As our item $l$, for each given $s \in[\alpha, \beta]$ all the sets $\underline{Y}_{0}^{j}(s, t, \underline{x}, \underline{u})(j \in\{1,2\})$ of active inequality constraints do not depend on the value of $t \in[a, b]$. Item 2 says, again for each given $s$, that all the feasible sets $\underline{M}_{s . t}^{u}$ are $C^{4}$-diffeomorphic for different values of $t \in[a, b]$. Moreover, item 3, whenever a trajectory runs through a generalized critical (or a Kuhn-Tucker) point then this generalized critical (or Kuhn-Tucker) trajectory entirely consists of generalized critical (Kuhn-Tucker) points (respectively). Here we use the chain rule. Item 4 is given in the sequel.

Let a pair $(\bar{x}, \bar{u}) \in\left(C^{4}([a, b], \mathbb{R})\right)^{n} \times\left(C^{4}([\alpha, \beta] \times[a, b], \mathbb{R})\right)^{q}$ be locally optimal, that is, a local minimum with respect to a topology to be precisely stated later, for the following problem of optimal control $\mathscr{P}_{\mathscr{S P}}(s)(s \in[\alpha, \beta]$ being given) in which $u$, jointly with $x$, is in competition, too. Then $\bar{x}$ is locally optimal, that is, a local minimum, for $\mathscr{P}_{\mathscr{S} \mathscr{S}}^{\bar{I}}(s)$ with the given control variable $\bar{u}(s, \cdot)$. Moreover, provided that for the feasible set the subsequently introduced constraint qualification (the extended Mangasarian-Fromovitz constraint qualification) holds, we learn from optimization (see $[21,36]$ ) that $\bar{x}$ is a Kuhn-Tucker trajectory and, hence, a generalized critical trajectory.

DEFINITION 3. Let $s \in[\alpha, \beta], t \in[a, b], C^{4}$-state and -control variables $x$ and $u$, respectively, with $x \in M_{s}^{\prime \prime}$, and $\underline{x} \in \underline{M}_{s, t}^{\prime \prime}, \underline{u}=u(s, t)$ be given. 
(a) (i) The Extended Linear Independence Constraint Qualification is said to hold for $\mathscr{P}_{\mathscr{S} \mathscr{I}}^{\prime \prime}(s, t)$ at $(t, \underline{x})$, if the conditions $\operatorname{ELI}_{s}^{u}, 2_{s}^{\prime \prime}$ are fulfilled.

ELI1: The set of active inequality constraints

$$
\underline{Y}^{\prime \prime}(s, t, \underline{x}):=\underline{Y}^{1}(s, t, \underline{x}, \underline{u}) \biguplus \underline{Y}^{2}(s, t, \underline{x}, \underline{u})
$$

(disjoint union) is finite.

ELI2: . The vectors $D_{\underline{x}} \underline{h}_{i}^{u}(s, t, \underline{x}), i \in \underline{I}$,

$$
\left.D_{\underline{\underline{x}}} \underline{g}_{j}^{u}(s, t, \underline{x}, \underline{y}, \underline{\xi})\right)\left((\underline{y}, \xi) \in \underline{Y}_{0}^{j}(s, t, \underline{x}, \underline{u},(\underline{y}, \xi)), \quad j \in\{1,2\}\right)
$$

are linearly independent.

(ii) The Extended Mangasarian-Fromovitz Constraint Qualification is said to hold for $\underline{P}_{\mathscr{S} \mathscr{S}}^{\prime \prime}(s, t)$ at $(t, \underline{x})$, if the conditions EMF1" $1_{s}^{u}, 2_{s}$ are fulfilled.

EMF1" The vectors $D_{\underline{x}} \underline{h}_{i}^{u}(s, t, \underline{x}), i \in \underline{I}$, are linearly independent.

$\mathbf{E M F 2}_{\mathrm{s}}^{\mathrm{u}}$. There exists a vector $\underline{\zeta}=\underline{\zeta}_{(s, t, \underline{x})}^{\prime \prime} \in \mathbb{R}^{n}$ satisfying

$$
\left\{\begin{aligned}
D_{\underline{x}} \underline{h}_{i}^{\prime \prime}(s, t, \underline{x}) \underline{\zeta}=0 & (i \in \underline{I}) \\
\left.D_{\underline{x}} \underline{g}_{j}^{\prime \prime}(s, t, \underline{x}, \underline{y}, \underline{\xi})\right) \underline{\zeta}>0 & \left((\underline{y}, \underline{\xi}) \in \underline{Y}_{0}^{j}(s, t, \underline{x}, \underline{u}), j \in\{1,2\}\right) .
\end{aligned}\right.
$$

Such a vector $\zeta$ is called an EMF-vector.

(b) The Extended Linear Independence Constraint Qualification and the MangasarianFromovitz Constraint Qualification, are said to hold for $\mathscr{P}_{\mathscr{S} \mathscr{I}}^{\prime \prime}(s)$ at the trajectory $x$ if there is a time $t^{\prime} \in[a, b]$ with $\underline{x}=x\left(t^{\prime}\right)$ and with the extended linear independence constraint qualification or the extended Mangasarian-Fromovitz constraint qualification (respectively) holding for $\underline{P}_{\mathscr{S} \mathscr{S}}^{\prime \prime}\left(s, t^{\prime}\right)$ at $\left(t^{\prime}, \underline{x}\right)$.

As our item 5 we state now that the extended linear independence constraint qualification is a stronger condition than the extended Mangasarian-Fromovitz constraint qualification. For further investigations of these and for the following conditions in the context of optimization we refer to $[33,40,41,64]$.

The conditions which are introduced next, contribute to the notions given in the Definitions 1 and 2.

DEFINITION 4. Let $s \in[\alpha, \beta], t \in[a, b], C^{4}$-state and -control variables $x$ and $u$, respectively, $\underline{x} \in \underline{M}_{s . t}^{\prime \prime}, \underline{u}=u(s, t)$ and $j \in\{1,2\}$ be given.

(a) (i) A point $(\underline{y}, \xi) \in \underline{Y}^{1}(s, t, \underline{x}, \underline{u})$ is called a critical point for the minimization problem of $\underline{g}_{1}^{\prime \prime}(s, t, \underline{x}, \cdot)$ on $\underline{Y}^{\prime}(s, t, \underline{x}, \underline{u})$ if both the linearized 
independence constraint qualification, that is, the extended linear independence constraint qualification for finite optimization, holds and there exist real numbers $\underline{\lambda}_{i}^{!}\left(i \in A^{\prime}\right), \underline{\mu}_{k}^{\prime}\left(k \in B_{0}^{\prime}(s, t, \underline{x}, \underline{u},(\underline{y}, \xi))\right)$, called Lagrange multipliers satisfying

$$
\begin{aligned}
& D_{(\underline{y}, \xi) \underline{g}_{1}^{u}}(s, t, \underline{x},(\underline{y}, \xi))=\sum_{i \in A^{\prime}} \underline{\lambda}_{i}^{l} D_{(\underline{y} . \xi)} \underline{\hat{h}}_{i}^{l, u}(s, t, \underline{x},(\underline{y}, \xi)) \\
& +\sum_{\ell \in B_{0}^{1}(s, t, \underline{\underline{x}} \underline{\underline{u} .,(y, \xi))}} \underline{\mu}_{\ell}^{1} D_{(\underline{y}, \xi)} \underline{\hat{g}}_{\ell}^{1 . u}(s, t, \underline{x},(\underline{y}, \xi)) .
\end{aligned}
$$

Here, we refer to the set of active inequality constraints at the feasible point $(\underline{y}, \xi)$ :

$$
\left.B_{0}^{\prime}(s, t, \underline{x}, \underline{u},(\underline{y}, \xi)):=\left\{\ell \in\left\{1, \ldots,\left|B^{\prime}\right|+2\right\} \mid \underline{\hat{g}}_{\ell}^{1 \cdot u}(s, t, \underline{x}, \underline{y}, \xi)\right)=0\right\} .
$$

(ii) A point $(\underline{y}, \xi) \in \underline{Y}^{2}(s, t, \underline{x}, \underline{u})$ is called a critical point for the minimization problem of $\underline{g}_{2}^{\prime \prime}(s, t, \underline{x}, \cdot)$ on $\underline{Y}^{2}(s, t, \underline{x}, \underline{u})$ if $\xi=a$, and if both the linearized independence constraint qualification holds and there exist Lagrange multipliers $\underline{\lambda}_{i}^{2}\left(i \in A^{2}\right), \underline{\mu}_{k}^{2}\left(k \in B_{0}^{2}(s, t, \underline{x}, \underline{u},(\underline{y}, a))\right)$, satisfying

$$
\begin{aligned}
& \left.D_{\underline{y}} \underline{g}_{2}^{u}(s, t, \underline{x},(\underline{y}, a))=\sum_{i \in A^{2}} \underline{\lambda}_{i}^{2} D_{\underline{y}} \underline{\hat{h}}_{i}^{2, u}(s, t, \underline{x}, \underline{y}, a)\right)
\end{aligned}
$$

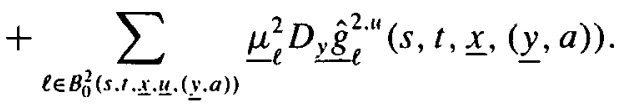

Here, we refer to the set of active inequality constraints at the feasible point $(\underline{y}, a)$ :

$$
\left.\left.B_{0}^{2}(s, t, \underline{x}, \underline{u}, \underline{y}, a)\right):=\left\{\ell \in B^{2} \mid \underline{\hat{g}}_{\ell}^{2 . u}(s, t, \underline{x}, \underline{y}, a)\right)=0\right\} .
$$

(iii) Let $(\underline{y}, \xi) \in \underline{Y}^{j}(s, t, \underline{x}, \underline{u})$ be a critical point with Lagrange multipliers $\left.\underline{\lambda}_{i}^{j}\left(i \in A^{j}\right), \underline{\mu}_{h}^{j}\left(k \in B_{0}^{j}(s, t, \underline{x}, \underline{u}, \underline{y}, \underline{\xi})\right)\right)$.

We define the Lagrange function $\underline{\hat{L}}^{j . u}$ as

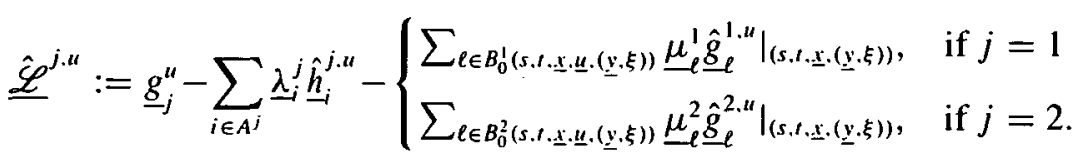

The critical point $(\underline{y}, \xi)$ is called nondegenerate if the following conditions hold. 
ND1
ND2.
ND.

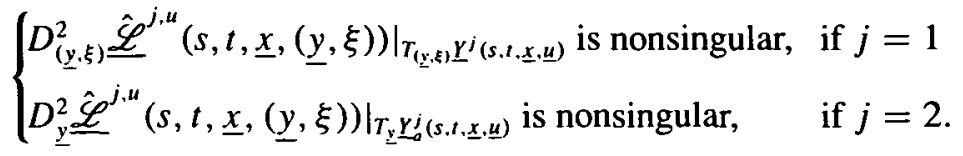

Here $T_{(\underline{y}, \xi)} \underline{Y}^{\prime}(s, t, \underline{x}, \underline{u})$ denotes the tangent space of $\underline{Y}^{\prime}(s, t, \underline{x}, \underline{u})$ at $(\underline{y}, \xi)$, that is,

$$
\begin{aligned}
& T_{(\underline{y}, \xi)} \underline{Y}^{\prime}(s, t, \underline{x}, \underline{u}):=\left\{\underline{\zeta} \in \mathbb{R}^{p+1} \mid D_{(\underline{y}, \xi)} \underline{\hat{h}}_{i}^{1, u}(s, t, \underline{x},(\underline{y}, \underline{\xi})) \underline{\zeta}=0\right. \\
& \left(i \in A^{1}\right) \text {, }
\end{aligned}
$$

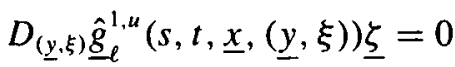

$$
\begin{aligned}
& \left.\left(\ell \in B_{0}^{1}(s, t, \underline{x}, \underline{u},(\underline{y}, \underline{\xi}))\right)\right\} \text {. }
\end{aligned}
$$

Moreover, $\left.D_{(\underline{y}, \xi)}^{2} \underline{\mathscr{L}}^{1, u}\right|_{T_{(\underline{\underline{y}}, \xi}, \underline{Y}^{1}(s, t, \underline{x}, \underline{u})}$ stands pointwise for the matrix $V^{T} D_{(\underline{y}, \xi)}^{2} \underline{\mathscr{L}}^{1, u} V$, where $V$ is some matrix of $(p+1)$-vectors which form a basis for the tangent space $T_{(\underline{y}, \xi)} \underline{Y}^{1}(s, t, \underline{x}, \underline{u})$.

Finally, $\left.D_{\underline{y}}^{2} \underline{\mathscr{L}}^{2, u}\right|_{T_{\underline{y}} \underline{Y}_{s}^{2}(s, t, \underline{x}, \underline{u})}$ is understood in the same sense, with $p+1$ replaced by $p$ and with $\underline{Y}_{a}^{2}(\cdot)$ defined by $\underline{Y}_{a}^{2}(\cdot) \times\{a\}=\underline{Y}^{2}(\cdot)$.

A critical point is called a nondegenerate Kuhn-Tucker point or a nondegenerate local minimum if it is both a nondegenerate critical point, and a Kuhn-Tucker point or a local minimum, respectively.

(b) The Reduction-Ansatz holds for the optimization problem $\underline{P}_{\mathscr{S} \mathscr{S}}^{u}(s, t)$ at $(t, \underline{x})$ if each index $(\underline{y}, \xi) \in \underline{Y}_{0}^{1}(s, t, \underline{x}, \underline{u}) \biguplus \underline{Y}_{0}^{2}(s, t, \underline{x}, \underline{u})$ is a nondegenerate as a local minimum of $\underline{g}_{1}^{u}(s, t, \underline{x}, \cdot)$ or $\underline{g}_{2}^{u}(s, t, \underline{x}, \cdot)$, respectively.

For this definition which refers to optimization problems of the lower stage the following item 6 holds. If the point $(y, \xi) \in \underline{Y}^{j}(s, t, \underline{x}, \underline{u})$ is a critical point or a nondegenerate critical point, a nondegenerate Kuhn-Tucker point or a nondegenerate local minimum then, with $s$ remaining fixed ands moving with $(t, \underline{x}), \underline{x}=x(t)$, along the flow $\Phi^{u},(\underline{y}, \xi) \in \underline{Y}^{j}(s, t, \underline{x}, \underline{u})$ has the same property at any other time $t^{\prime} \in[a, b]$, respectively. This fact is based on the definition of the data on the lower stage, with $\Phi^{u}$ being inserted. Moreover, because of a similar reasoning, the definition of the Reduction-Ansatz is $t^{\prime}$-independent in the same sense, too. This means that the Reduction-Ansatz holds with respect to a given local minimum $(\underline{y}, \xi)$ either at no $t^{\prime} \in[a, b]$ or at each $t^{\prime} \in[a, b]$. Hence, we might refer to the Reduction-Ansatz for the optimal control problem $\mathscr{P}_{\mathscr{S}_{\mathscr{S}}^{u}}^{u}(s)$ at $x$ where $x$ is a given feasible trajectory. 
CONVENTION 1. The previous introduction of Lagrange functions $\underline{\hat{L}}^{j . \prime \prime}$ gives rise to a further generalization of our different critical point conditions or constraint qualifica. tions (Definitions 1-4). Namely, we replace $g_{j}^{\prime \prime}$ by $\underline{\mathscr{L}}^{j . \prime \prime}$.

If the Reduction-Ansatz holds at $(t, \underline{x}), \underline{x} \in \underline{M}_{s, l}^{u}$, with respect to the parameter $s$ then we get the opportunity locally around $(s, t, \underline{x})$ and around an active index $(\underline{y}, \xi)$ to represent each of the corresponding local minima $(\underline{\tilde{y}}, \tilde{\xi})$ as an implicit function $(\underline{\tilde{y}}, \tilde{\xi})=\left(y^{\prime \prime}(s, t, \underline{x}), \xi^{\prime \prime}(s, t, \underline{x})\right)$. Then, near $(\underline{y}, \underline{\xi})$ we have, in essence, only on inequality constraint defined by a marginal function ( $c f$. [67]):

$$
\underline{g}^{\mathscr{U} \cdot u}(s, t, \underline{x}):=\underline{g}_{j}^{u \prime}\left(s, t, \underline{x}, y^{\prime \prime}(s, t, \underline{x}), \xi^{\prime \prime}(s, t, \underline{x})\right) \geq 0
$$

(here, $\xi^{\prime \prime}$ is the constant function $a$, if $j=2$ ). This reduction always leads us to a locally defined problem $\underline{P}_{\mathscr{F}}^{\mathscr{U}} \cdot$ " $(s, t)$ called a problem of the upper stage.

Of course, the notions "(nondegenerate) critical", "(nondegenerate) Kuhn-Tucker' and "(nondegenerate) local minimal", as given above for $j=1$, can also be introduced for problems $\underline{P}_{\mathscr{F}}^{\mathscr{Z}} \cdot \|(s, \bar{t})$, and hence $\mathscr{P}_{\mathscr{S} \mathscr{\mathscr { S }}}^{\prime \prime}(s)$. Therefore they also lead us to the notions of a (nondegenerate) critical trajectory, and, in particular, of a nondegenerate KuhnTucker trajectory and of a nondegenerate local minimum trajectory. Note, that for a point or a trajectory being critical is a weaker condition than being "Kuhn-Tucker", but a stronger condition than being "generalized critical".

Our stability and genericity investigations in spaces of functions are based on the following topologies. The topology $\left(C_{S}^{3}, C_{S}^{4}\right)$ for the product $\left(\prod_{i=1}^{r_{1}} C^{3}\left(\mathscr{M}_{i}, \mathbb{R}\right)\right) \times$ $\left(\prod_{j=r_{1}+1}^{r_{2}} C^{4}\left(\mathscr{M}_{j}, \mathbb{R}\right)\right)$, with $\mathscr{M}_{k}$ being some $\mathbb{R}^{\ell}$ or $[\alpha, \beta] \times[a, b]$, will be the producttopology generated by the strong (or Whitney-) $C^{d}$-topology $C_{S}^{d}$ on each factor $C^{d}\left(\mathscr{M}_{k}, \mathbb{R}\right)(d \in\{3,4\}$, respectively; $c f .[22,28])$. As for $d=3$ we need only to refer to the derivatives up to order 3 , and for $d \in\{0,1,2\}$ correspondingly, let us consider $d=4$. A typical base-neighbourhood of a function $\rho \in C^{4}\left(\mathscr{M}_{j_{0}}, \mathbb{R}\right)\left(j_{0} \in\right.$ $\left.\left\{r_{1}+1, \ldots, r_{2}\right\}\right)$ is the set $\rho+\mathscr{W}_{\epsilon}$, where $\mathscr{W}_{\epsilon}$ is defined with the aid of a controlling continuous positive function $\epsilon: \mathscr{M}_{j_{0}} \rightarrow \mathbb{R}$ :

$$
\begin{aligned}
\mathscr{W}_{\epsilon}:=\left\{\eta \in C^{4}\left(\mathscr{M}_{j_{0}}, \mathbb{R}\right) \mid\right. & |\eta(\underline{\omega})|+\sum_{i}\left|\frac{\partial \eta}{\partial \omega_{i}}(\underline{\omega})\right| \\
& +\sum_{i, j}\left|\frac{\partial^{2} \eta}{\partial \omega_{i} \partial \omega_{j}}(\underline{\omega})\right|+\sum_{i, j . k}\left|\frac{\partial^{3} \eta}{\partial \omega_{i} \partial \omega_{j} \partial \omega_{k}}(\underline{\omega})\right| \\
& \left.+\sum_{i, j . k, \ell}\left|\frac{\partial^{4} \eta}{\partial \omega_{i} \partial \omega_{j} \partial \omega_{k} \partial \omega_{\ell}}(\underline{\omega})\right|<\epsilon(\underline{\omega}) \text { for all } \underline{\omega} \in \mathscr{M}_{j_{0}}\right\}^{(4)} .
\end{aligned}
$$

Note, that in the case $\mathscr{M}_{j_{0}}=[\alpha, \beta] \times[a, b]$ we may equivalently concentrate on al $]$ constant controlling $\epsilon$, while in the case $\mathscr{M}_{j_{0}}=\mathbb{R}^{\ell}$ the variability of $\epsilon(\underline{\omega})$ allows us tc take asymptotic effects into account when $|\underline{\omega}|$ goes to infinity. 


\section{Generic types, main results}

Following the general presentation in [20] let us now give a short introduction of the eight types of generalized critical points $\underline{\bar{x}} \in \underline{M}_{\bar{s} . \bar{u}}^{\bar{u}}(\bar{s} \in[\alpha, \beta], \bar{t} \in[a, b])$ which may later be called "generic" corresponding to the generalized critical points. They have in common that their degeneracy at the upper or at the lower stage, if ever such a degeneracy is allowed, is as slight as possible. Examples will be given in the next section with the normal forms there.

From Assumption 1 and with a continuity argument it follows that the set-valued mapping $\underline{Y}^{\prime}(s, t, \underline{x}, \underline{u}) \biguplus \underline{Y}^{2}(s, t, \underline{x}, \underline{u})$ is upper semi-continuous and has compact values. This condition may be our Assumption 1 here.

We distinguish three groups of generalized critical points.

Group I (Types 1-5). At $\underline{\bar{x}}$ the Reduction-Ansatz holds for $\underline{P}_{\mathscr{S} \mathscr{S}}^{\bar{u}}(\bar{s}, \bar{t})$. With this fact and with the implicit function theorem whose application is based on the Reduction-Ansatz, we conclude that the set

$$
\underline{Y}_{0}^{\bar{u}}(\bar{s}, \bar{t}, \underline{\bar{x}}):=\underline{Y}_{0}^{1}(\bar{s}, \bar{t}, \underline{\bar{x}}, \underline{\bar{u}}) \biguplus \underline{Y}_{0}^{2}(\bar{s}, \bar{t}, \underline{\bar{x}}, \underline{\bar{u}}) \quad(\underline{\bar{u}}=\bar{u}(\bar{s}, \bar{t}))
$$

has at most a finite number of elements, say $\kappa$, that is, there are nonnegative integers $\kappa_{1}, \kappa_{2}$ with $\kappa=\kappa_{1}+\kappa_{2}$ and with

$$
\begin{gathered}
\underline{Y}_{0}^{\prime}(\bar{s}, \bar{t}, \bar{x}, \bar{u})=\left\{\left(\underline{y}^{1}, \xi^{\prime}\right), \ldots,\left(\underline{\left.\left.y^{\kappa_{1}}, \xi^{\kappa_{1}}\right)\right\},}\right.\right. \\
\underline{Y}_{0}^{2}(\bar{s}, \bar{t}, \underline{\bar{x}}, \underline{\bar{u}})=\left\{\left(\underline{y^{\kappa_{1}+1}}, \xi^{\kappa_{1}+1}\right), \ldots,\left(\underline{y^{\kappa}}, \xi^{\kappa}\right)\right\} \quad\left(\xi^{\ell}=a, \ell \in\left\{\kappa_{1}+1, \ldots, \kappa\right\}\right) .
\end{gathered}
$$

In the way of (3), locally around $\bar{s}, \bar{t}, \underline{\underline{x}},(y, \xi)$, say, especially, in a neighbourhood $\mathscr{U}=\mathscr{U}_{\bar{s}} \times \mathscr{U}_{\bar{t}} \times \mathscr{U}_{\underline{\underline{x}}}$ of $(\bar{s}, \bar{t}, \underline{\bar{x}})$ we introduce the marginal functions $\underline{g}_{j . \ell}^{\mathscr{U} . \bar{u}}(s, t, \underline{x})((s, t$, $\left.\underline{x}) \in \mathscr{U}, \ell \in\left\{1, \ldots, \kappa_{j}\right\}, j \in\{1,2\}\right)$. Then we introduce the types $1-5$ for the generalized critical point of our given semi-infinite optimization problem referring to the following finite optimization problem. Locally, in $\mathscr{U}$, both problems are equivalent. We consider the functions

$$
\underline{G}^{\mathscr{U} \cdot \bar{u}}:=\left(\underline{g}_{1}^{\mathscr{U} \cdot \bar{u}}, \underline{g}_{2}^{\mathscr{U} \cdot \bar{u}}\right)=\left(\left(\underline{g}_{1 . \ell}^{\mathscr{U} \cdot \bar{u}}\right)_{\ell \in\left\{1 \ldots . \kappa_{1}\right\}},\left(\underline{g}_{2 . \ell}^{\mathscr{U} \cdot \bar{u}}\right)_{\ell \in\left\{1 \ldots . \kappa_{2}\right\}}\right)
$$

and the finite problem

$$
\begin{aligned}
& \underline{P}_{\mathscr{F}}^{\mathscr{U} \cdot \bar{u}}(\bar{s}, \bar{t}):=\underline{P}_{\mathscr{F}}^{\mathscr{U}}\left(\underline{f}^{\bar{u}}(\bar{s}, \bar{t}, \cdot), \underline{H}^{\bar{u}}(\bar{s}, \bar{t}, \cdot), \underline{G}^{\mathscr{U} \cdot \bar{u}}(\bar{s}, \bar{t}, \cdot)\right), \\
& \left\{\begin{array}{l}
\operatorname{Min} f^{\bar{u}}(\bar{s}, \bar{t}, \cdot) \text { on } \underline{M}_{\bar{s}, \bar{u}}^{\mathscr{U}, \bar{u}}, \text { where } \\
\underline{M}_{\bar{s}, \bar{u}}^{\mathscr{U} . \bar{u}}:=\underline{M}_{\bar{s}, \bar{t}}^{\bar{u}} \cap \mathscr{U}_{\underline{\underline{x}}}=\left\{\underline{x} \in \mathscr{U}_{\overline{\underline{x}}} \mid\right. \\
\left.\underline{h}_{i}^{\bar{u}}(\bar{s}, \bar{t}, \underline{x})=0(i \in \underline{I}), \underline{g}_{j . \ell}^{\mathscr{U} \cdot \bar{u}}(\bar{s}, \bar{t}, \underline{x}) \geq 0 \quad\left(\ell \in\left\{1, \ldots, \kappa_{j}\right\}, j \in\{1,2\}\right)\right\} .
\end{array}\right.
\end{aligned}
$$


Moreover, because of the equations ( $c f$. [64])

$$
D_{\underline{x}} \underline{g}_{k}^{\bar{u}}\left(\bar{s}, \bar{t}, \bar{x},\left(\underline{y}^{\ell}, \xi^{\ell}\right)\right)=D_{\underline{x}} \underline{g}_{k, \ell}^{\mathscr{U}, \bar{u}}(\bar{s}, \bar{t}, \underline{\bar{x}}) \quad(k \in\{1,2\})
$$

we may for the (locally defined) finite and for the semi-infinite problem refer to the same multipliers due to a generalized critical point (or due to a Kuhn-Tucker point, as Lagrange multipliers). These were introduced in the Definitions 1 (a) and 2 (a) and may for the inequality constraints now also be called $\mu_{j}:=\mu_{k . \ell}\left(j=\ell \in\left\{1, \ldots, \kappa_{1}\right\}\right.$, if $k=1 ; j=\kappa_{1}+\ell, \ell \in\left\{1, \ldots, \kappa_{2}\right\}$, if $k=2$ ).

For the locally defined Lagrange function of the problem $\underline{P}_{\mathscr{F}}^{\mathscr{U}} \overline{\bar{u}}(\bar{s}, \bar{t})$ we write $\underline{\mathscr{L}}^{\mathscr{U} \cdot \bar{u}}(\bar{s}, \bar{t}, \underline{x})$.

A precise definition of these five types is given in $[29,30]$ referring to transversality theory ( $c f$. also [22, 28, 62, 69]). Here we may give a rough description essentially pointing out for the types $2-5$ the difference with type 1 . The third order derivatives which we shall demand for the optimization problem, are only motivated by type 3 .

Type 1. Our point $\bar{x} \in M_{\bar{s}, \bar{t}}^{\bar{u}}$ is a nondegenerate critical point, in the sense of Definition 4 (a)(iii), of $\underline{P}_{\mathscr{F}}^{\mathscr{U}, \bar{u}}(\bar{s}, \bar{t})$ (in particular, the linearized independence constraint qualification is satisfied).

Type 2. The linearized independence constraint qualification is satisfied, and exactly one of the (because of the linearized independence constraint qualification uniquely determined) Lagrange multipliers $\mu_{j}$ vanishes.

Type 3. The linearized independence constraint qualification is satisfied, and exactly one eigenvalue $\sigma_{\ell}$ of the matrix $\left.D_{\underline{x}} \underline{L}^{\mathscr{U} \cdot \bar{u}}\right|_{T_{\underline{\underline{T}}} \underline{M}_{\bar{i}, \bar{i}}^{\mathscr{U}} \bar{i}}$ at $(\bar{s}, \bar{t}, \bar{x})$, vanishes.

Type 4. The linearized independence constraint qualification is violated, and the number of active constraints satisfies the inequality $|\underline{I}|+\left|\underline{Y}_{0}^{\bar{u}}(\bar{s}, \bar{t}, \underline{\bar{x}})\right| \leq n$.

It turns out that the version of the extended Mangasarian-Fromovitz constraint qualification for finite optimization, the Mangasarian-Fromovitz constraint qualification, is violated as well.

Type 5. The linearized independence constraint qualification is violated, and the number of active constraints satisfies the equality $|\underline{I}|+\left|\underline{Y}_{0}^{\bar{u}}(\bar{s}, \bar{t}, \bar{x})\right|=n+1$. Then the Mangasarian-Fromovitz constraint qualification might be satisfied or not.

For those types which belong to the groups II or III the Reduction-Ansatz is violated. Let us indicate the characteristic features of these types.

\section{Group II (Type 6)}

(a) At all points $(\underline{y}, \xi) \in \underline{Y}_{0}^{\vec{u}}(\bar{s}, \bar{t}, \bar{x})$ the linearized independence constraint qualification condition is satisfied at $(y, \xi)$.

(b) Exactly one point $\left(y^{*}, \xi^{*}\right) \in \underline{Y}_{0}^{\bar{u}}(\bar{s}, \bar{t}, \bar{x})$ is a degenerate local minimum for $\underline{g}_{k}^{\bar{u}}(\bar{s}, \bar{t}, \underline{\bar{x}}, \cdot)$ over $\underline{Y}_{0}^{k}(\bar{s}, \bar{t}, \underline{\bar{x}}, \underline{\bar{u}})$ with $k=1$ or $k=2$. The degeneracy consists 
in the vanishing of exactly one Lagrange multiplier corresponding to an active inequality constraint.

Note that here the particular degeneracy on the lower stage is known from type 2 on the upper stage. A precise characterization of a point of type 6 , essentially by means of transversality conditions, is given in [20]. The types 7,8 of the following last group are in detail studied in [35].

\section{Group III (Types 7, 8)}

(a) At exactly one point $\left(\underline{y}^{*}, \xi^{*}\right) \in \underline{Y}_{0}^{\bar{u}}(\bar{s}, \bar{t}, \underline{x})$, say $\left(y^{*}, \xi^{*}\right) \in \underline{Y}_{0}^{k^{*}}(\bar{s}, \bar{t}, \underline{\bar{x}}, \underline{\bar{u}}), k^{*} \in$ $\{1,2\}$, the linearized independence constraint qualification condition is violated. All other points from $\underline{Y}_{0}^{\bar{u}}(\bar{s}, \bar{t}, \underline{\bar{x}})$ are nondegenerate local minima for $\underline{g}_{k}^{\bar{u}}(\bar{s}, \bar{t}, \underline{\bar{x}}, \cdot)$ over $\underline{Y}_{0}^{k}(\bar{s}, \bar{t}, \underline{\bar{x}}, \underline{\bar{u}})$ with $k=1$ or $k=2$, respectively.

(b) Type 7: $\left|A^{k^{*}}\right|+\left|B_{0}^{k^{*}}\left(\bar{s}, \bar{t}, \underline{\underline{x}}, \underline{\underline{u}},\left(\underline{y^{*}}, \xi^{*}\right)\right)\right| \leq \begin{cases}p+1, & \text { if } k^{*}=1 \\ p, & \text { if } k^{*}=2\end{cases}$

Type 8: $\left|A^{k^{*}}\right|+\left|B_{0}^{k^{*}}\left(\bar{s}, \bar{t}, \underline{\bar{x}}, \underline{\underline{u}},\left(\underline{y^{*}}, \xi^{*}\right)\right)\right|= \begin{cases}p+2, & \text { if } k^{*}=1 \\ p+1, & \text { if } k^{*}=2 .\end{cases}$

REMARK 3. For each $t \in[a, b]$ the points of type $2-8$ form a discrete subset of $\underline{\Sigma}_{g c}(t)$ (cf. $[20,30])$.

We say that a generalized critical trajectory $\bar{x}$ is of one of the types $1-8$ if for some time $t \in[a, b]$ the point $\underline{x}=\bar{x}(t)$ is a generalized critical point of the corresponding optimization problem. Moreover, we state the following item which can be proved as the related items 1-6 from Section 2. Namely, a generalized critical (or Kuhn-Tucker) trajectory of one of the types $1-8$ does exclusively contain generalized critical (or Kuhn-Tucker) points precisely of this type.

Having turned from optimal control to optimization, for the moment we disregard the fact that we generated our optimization problem from the given optimal control problem in the way given by (la), (1b). In particular, $\bar{u}$ becomes suppressed. Furthermore, let us denote the variable $t \in[a, b]$ which we shall keep fixed, as an index, for example, writing $\underline{f}(s, \underline{x}):=\underline{f}(s, t, \underline{x})$. However, we look at the optimization problem as another topological object of interest. So we let the $C^{3}$-functions defining the optimization problem vary such that we get the class of three times continuously differentiable semi-infinite optimization problems of given form and dimensions. Up to Assumption 1, now, we ask due to all these problems whether each of their generalized critical points is of one of the eight types.

NOTE 1 . For each $t \in[a, b]$ we set

$$
\underline{\operatorname{CUSC}}(t):=\left\{\left(\underline{g}_{2 i}, \ldots, \underline{\hat{h}}_{i t}^{1}, \ldots, \underline{\hat{h}}_{i t}^{2}, \ldots, \underline{\hat{g}}_{j t}^{1}, \ldots, \underline{\hat{g}}_{j t}^{2}, \ldots\right) \mid\right.
$$


Assumption 1 holds for the set-valued mapping corresponding to $\left(\underline{\widehat{H}}_{t}, \underline{\widehat{G}}_{t}\right) ; \underline{g}_{2 t}, \hat{\hat{h}}_{i t}^{2}\left(i \in A^{2}\right), \underline{\hat{g}}_{j t}^{2}\left(j \in B^{2}\right)$ do not depend on $\left.\xi\right\}$.

A related set of data of our optimal control problems will be given below.

Now, we can both specify and, hence, almost directly apply [20], Theorem 2, within the context of semi-infinite optimization problems of the form $\underline{\mathscr{P}}\left(f^{\bar{u}}(s, t, \cdot),\left(\underline{H}^{\bar{u}}(s, t\right.\right.$, \left.\left..), \underline{G}^{\bar{u}}(s, t, \cdot)\right),\left(\widehat{\hat{H}}^{\bar{u}}(s, t, \cdot), \widehat{G}^{\bar{u}}(s, t, \cdot)\right)\right)$ such that we learn the following theorem on the general position of optimization problems with generalized critical points exclusively being of the types $1-8$. Indeed, hereby, we additionally have only to realize that the special functions $\underline{\hat{g}}_{\ell}\left(\ell \in\left\{\left|B^{1}\right|+1,\left|B^{1}\right|+2\right\}\right)$, say, with $\xi=a$ fixed, may remain unchanged. In the density part, here, on the lower stage, for example, we transversally perturb the (other) $\xi$-independent data $\underline{g}_{21}, \hat{h}_{i \prime}^{2}\left(i \in A^{2}\right), \underline{\hat{g}}_{\ell}(\ell \in$ $\left.\left\{\left|B^{1}\right|+3, \ldots,\left|B^{1}\right|+\left|B^{2}\right|+2\right\}\right)$. For an in part similar argumentation we may refer to $[64,65]$. Indeed, in $[64,65]$ because of the special form of a max-type objective function there is an invariant part played by its level parameter. Here, however, the auxiliary time variable $\xi$ is treated as an invariant of the defining functions on the lower stage.

THEOREM 1 (Genericity Theorem A). For each $t \in[a, b]$ there exists $a C_{S}^{3}$-open and -dense subset $F_{\mathscr{S} \mathscr{I}}(t)$ of $\left(C^{3}\left(\mathbb{R}^{n+1}, \mathbb{R}\right)\right)^{|!|+1} \times C^{3}\left(\mathbb{R}^{n+q+2}, \mathbb{R}\right) \times \underline{C U S C}(t)$ such that we have for all

$$
\left(\underline{f}_{i}, \ldots, \underline{h}_{i t}, \ldots, \underline{g}_{1 t}, \underline{g}_{2 t}, \ldots, \underline{\hat{h}}_{i t}^{1}, \ldots, \underline{\hat{h}}_{i t}^{2}, \ldots, \underline{\hat{g}}_{j t}^{1}, \ldots, \underline{\hat{g}}_{j t}^{2}, \ldots\right) \in F_{\mathscr{S P}}(t),
$$

each element of the (corresponding) generalized critical point set $\underline{\Sigma}_{g c}(t)$ is of one of the types $1-8$.

As a $C_{S}^{3}$-open and -dense subset $F_{\mathscr{S P}}(t)$ is in particular a generic subset of the underlying restricted space of $C^{3}$ - functions. By definition, a generic or residual subset needs (only) to contain the intersection of a sequence of open and dense subsets; $c f$. [22, 23, 28].

Now, the question raises to what an extent this result can be formulated due the varying functional data which define our optimal control problems. At first we write referring to the different sets, that is, Euclidean spaces or full rectangles, where our (functional) data are defined on:

$$
\text { CUSCLB }:=\left(\ldots, \hat{h}_{i}^{1}, \ldots, \hat{h}_{i}^{2}, \ldots, \hat{g}_{j}^{1}, \ldots, \hat{g}_{j}^{2}, \ldots, f_{k}, \ldots, u_{\ell}, \ldots\right) \mid
$$

the coordinate functions of $(\widehat{H}, \widehat{G}),(F, u)$ are $C^{3}-, C^{4}$-functions, respectively; Assumption $I$ holds for the set-valued mapping $Y^{j}=Y_{(\widehat{H} . \widehat{\sigma} . u)}^{j}, j \in\{1,2\}$, being 
defined due to $(\widehat{H}, \widehat{G}, u)$, and Assumption 2 holds for $(F, u)$ (with a suitable

$$
\left.C^{4} \text {-extension ŭ of } u \text { on } \mathbb{R} \times \mathbb{R}\right) \text { \}. }
$$

Next we note that the function which represents the generating the (functional) optimization problems' data from the (functional) optimal control problems' data is continuous ( $c f$. (1a), (1b)). Let us denote this flow projective generating by $\mathscr{G}$. As a reflection on the constant state functions $x$ called the steady states, where $F=0$, and on $(t, \underline{u})$-independent optimization data shows, this function $\mathscr{G}$ is surjective. However, $\mathscr{G}$ is not injective. Namely, for example, adding on $L$ of a function $\ell(t)$ which does not depend on $(\underline{x}, \underline{u})$ but which is symmetric with respect to the point $t=\frac{a+b}{2}$, does not change the values of the objective function. Namely, we note $\int_{a}^{b} \ell(t) d t=0$. In this sense it preserves the generated optimization problem.

Now, Theorem 1 and the properties of $\mathscr{G}$ imply that the class of optimal control problems whose generalized critical trajectories exclusively belong to the types $1-8$, is $C_{S}^{3}$-open in the underlying topological space of functions fulfilling Assumptions 1 and 2 .

Moreover, whenever our optimization problem for $t=a$, being generated by an optimal control problem, becomes slightly perturbed, namely in the $C_{S^{-}}^{4}$ and $C_{S}^{3}$-sense for the $C^{4}$-objective function and for the $C^{3}$-constraints, respectively, then it remains generated by a slightly $C_{S}^{3}$ and $C_{S}^{4}$-perturbed optimal control problem. Here for the optimal control problem $C_{S}^{4}$ precisely refers to the functional data $(F, u)$. In fact, the latter problem may be made arbitrarily $C_{S}^{3}$-close to the unperturbed optimal control problem. Compared with the causal and constructive generating $\mathscr{G}$ this calculation refers to the opposite direction of thinking. We may call it reconstruction. Here for the generating perturbation of the optimal control problem we do not even need to change the functional data $(F, u)$. Let us call the perturbing additional functions of the optimization problem $\left(\left(C_{S}^{3}, C_{S}^{4}\right)\right.$-)mixed variations.

For a better understanding of this reconstruction we state two items. The first item says that by one raised order of differentiability, $C^{4}$, both of $\delta f$ and of $(F, u)$, takes account of their partial derivatives in the (re)construction of the perturbed $C^{3}$-function $\widetilde{L}$. Indeed, as a difference the variation represents the perturbation $\underline{f}_{a}^{u} \rightarrow \underline{\tilde{f}}$ which is again caused by a small perturbation $L \rightarrow \widetilde{L}$ :

$$
\begin{gathered}
\widetilde{L}:=L+\delta L, \text { where } \\
(\delta L)(s, t, \underline{x}, \underline{u}):=-D_{\underline{x}}(\delta \underline{f})(s, \underline{x}) \frac{\partial}{\partial t} \Phi^{\prime \prime}(s, \underline{x}, 0)+\frac{1}{b-a}(\delta \underline{f})\left(s, \Phi^{\prime \prime}(s, \underline{x}, b-t)\right) .
\end{gathered}
$$

A small calculation actually shows: $\int_{a}^{b} \widetilde{L}(s, t, x(t), u(s, t)) d t=\underline{f}_{a}(s, x(a))+$ $(\delta \underline{f})(s, x(a))=\underline{f}(s, x(a))$. As the second item we remark that the reconstruction of the other data, namely of $\widetilde{H}, \widetilde{G}, \widetilde{\widehat{H}}, \widetilde{\widehat{G}}$, can be obtained by taking the corresponding perturbed data of the optimization problem along the flow. 
Now, we focus our attention on those mixed variations which are chosen by demands of transversality $[20,30]$, that is, which guarantee the trajectories belonging to type 1-8 and, hence, cause the desired $\left(C_{S}^{3}, C_{S}^{4}\right)$-density, up to our two assumptions.

Hence we have realized the following result.

THEOREM 2 (Genericity Theorem B). There exists a $\left(C_{S}^{3}, C_{S}^{4}\right)$-open and -dense subsel $F_{\mathscr{S} \mathscr{I}}$ of the space $\left(C^{3}\left(\mathbb{R}^{n+p+2}, \mathbb{R}\right)\right)^{|l|+1} \times C^{3}\left(\mathbb{R}^{n+p+q+2}, \mathbb{R}\right) \times\left(C^{3}\left(\mathbb{R}^{2(n+p)+3}, \mathbb{R}\right)\right)^{\left|l_{a}^{b}\right|} \times$ $\left(C^{3}\left(\mathbb{R}^{2(n+p)+q+3}, \mathbb{R}\right)\right)^{\left|J_{\Delta}^{b}\right|} \times C$ USCLB such that we have for all

$$
\begin{aligned}
& \left(L, \ldots, h_{i}, \ldots, G, \ldots,\left(h_{a}^{b}\right)_{i}, \ldots, G_{a}^{b}, \hat{h}_{i}^{1}, \ldots, \hat{h}_{i}^{2}, \ldots, \hat{g}_{j}^{1}, \ldots, \hat{g}_{j}^{2}, \ldots,\right. \\
& \left.f_{k}, \ldots, u_{\ell}, \ldots\right) \in F_{\mathscr{S P I}}
\end{aligned}
$$

each element of the generalized critical trajectory set $\Sigma_{g c}$ is of one of the types $1-8$.

REMARK 4. In Section 1 we indicated some directions in which our problem mode] can be extended such that the corresponding versions of the Theorems 1 and 2 remain true. We emphasize, in particular, that both genericity theorems remain true in the nondifferentiable case of objective functionals being of maximum type.

A comparison of Theorems 1,2 shows the qualitative difference between optimization and optimal control, consisting in Assumption 2 which guarantees integrability (controllability). In further research the author will analyze this "gap" by means of describing the part played by the topology of the flow, that is, of the homological structure $[27,61]$ of its phase portrait.

In Figure 1 the sets $\underline{\Sigma}_{g c}(t), \underline{\Sigma}_{K T}(t)$ are illustrated locally in the neighbourhood of a point of type $1-5$, respectively $(c f .[20,30])$. We note that the figures due to the types 2-5 do also give an imagination of the critical sets due to the problems of the lower stage, of minimizing the inequality constraint on its index manifold, corresponding to the types $6-8$, respectively.

This illustration refers to a given parameter $t$. For two different parameter values $t^{1}, t^{2} \in[a, b]$ the sets $\underline{\Sigma}_{g c}\left(t^{1}\right)$ (or $\left.\underline{\Sigma}_{K T}\left(t^{1}\right)\right)$ and $\underline{\Sigma}_{g c}\left(t^{2}\right)$ (or $\underline{\Sigma}_{K T}\left(t^{2}\right)$, respectively) are $C^{4}$-diffeomorphic under the family of corresponding flows $\Phi^{\prime \prime}(s, \cdot)(s \in[\alpha, \beta])$ which pointwise preserve the analytical information of our generalized critical (and Kuhn-Tucker) trajectories.

For example, it may be that there is a whole trajectory of generalized critical points of type 2 over all $t \in[a, b]$ which is caused by a single boundary effect belonging to the state constraint at one particular time $t_{0}$ or to a constraint of (time-)boundary value form.

Whenever we discuss the equivalent optimization problems at a parameter $s$, we may in the sequel refer to a fixed time $\bar{t}$, for example, to $\bar{t}=a$ or to any time $\bar{t} \in[a, b]$, with its corresponding problems being $\underline{P}_{\mathscr{S} \mathscr{S}}^{u}(s, \bar{t})(s \in[\alpha, \beta])$. We may sometimes 


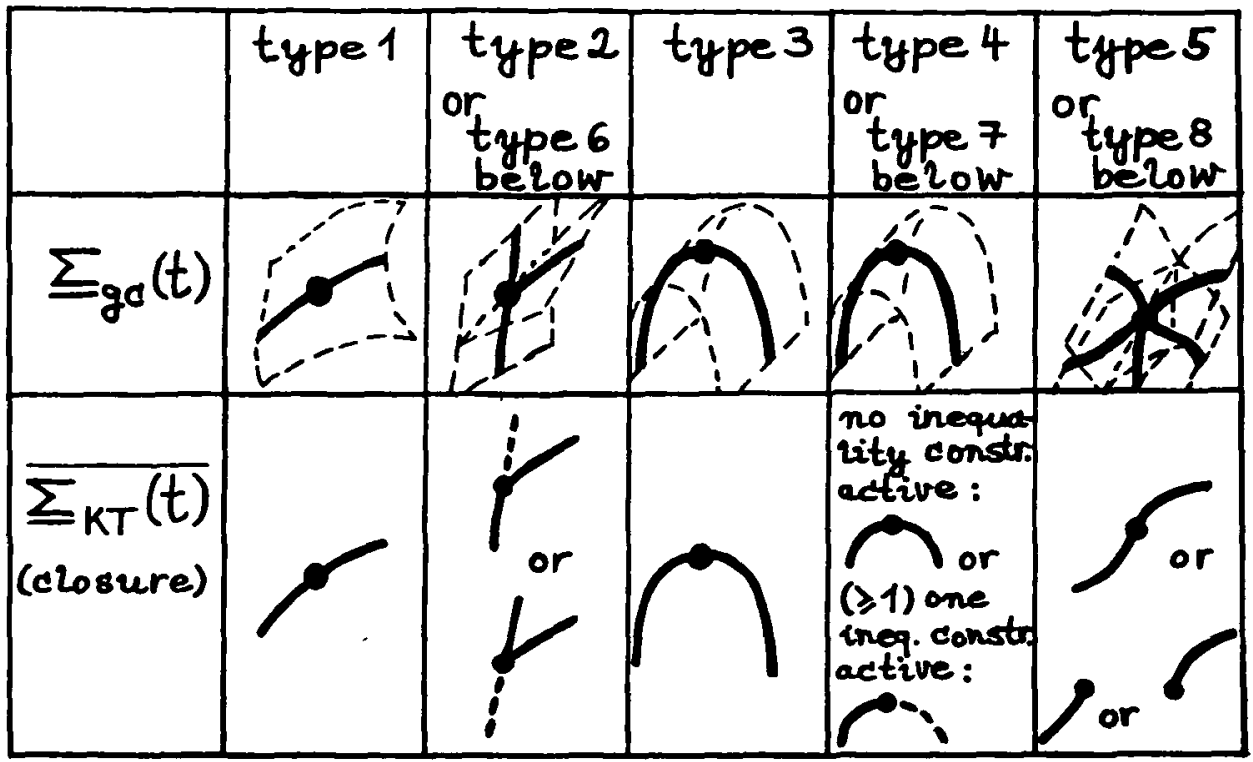

FIGURE 1. The optimization problem and the corresponding problem of the lower stage: $\underline{\Sigma}_{g r}(t), \underline{\Sigma}_{K T}(t)$ locally around a generalized critical or a Kuhn-Tucker point of a generic type $(t \in[a, b])$, and the embedding leaves.

for simplicity also suppress the notation of the $t$-dependence. Because of the flows $\Phi^{\prime \prime}(s, \cdot)$ our analytical and numerical expositions can be regarded as simultaneous (independent) with respect to $t \in[a, b]$. Correspondingly, the local critical curves and half curves in $\mathbb{B}^{n+1}$ which we shall look at for one time $t$, are embedded in local critical planes or half planes in $\mathbb{R}^{n+2}$ being due to all times $t$. The latter sets may be called leaves which can also be creased or pieced (see Figure 1). We emphasize that such a leaf $\mathscr{B}=\mathscr{B}_{g c}^{\prime \prime}$ in particular depends on $u$, and that it in a pointwise sense represents the set $\Sigma_{g c}$ containing the subset $\Sigma_{K T}$ as one or several "pieces".

Let us now study easy examples of normal forms of optimal control problems in the neighbourhood of our typical generalized critical points. Hereby we refer to integral functionals whose integrands do also depend on the derivative $\dot{x}$. Of course, $\dot{x}$ can formally be eliminated by substituting it with the right hand side of the differential equation. The power functions of the integrands will up to the factor -1 tum out to be "normal forms" $\mathscr{N} \mathscr{F}$ (at time $t$ ) of objective functions in optimization. Now, as the differential equation is suitably chosen, the integral functional can be expressed as half the normal form in the sense of optimization, $\frac{1}{2} \mathscr{N} \mathscr{F}$ evaluated at $\underline{x}:=x(a)$.

NOTE 2. Any other differential equation which fulfills Assumption 2, in particular all linear(ized) differential equations, could be chosen, too. As an example, we might 
consider

$$
\dot{x}=A(s, t) x+B(s, t) u(s, t) .
$$

For a suitable qualitative theory of nonlinear differential equations, their normal forms, which can be used in the $\underline{x}$-space $\mathbb{R}^{n}$ or in the $(t, \underline{x})$-space $\mathbb{R}^{n+1}$ and for their structural frontiers, we refer to the different approaches in $[2,25]$.

Until now, we have used the notion of a normal form in the meaning of an easy example of the objective function(al) or of the whole problems, parametrized by $s$. In the case of several types the objective functions and the constraints of the optimization problem at time $a$, which is equivalent with the given optimal control problem, constitute a "normal form" in the sense of the following proper meaning.

The underlying optimization data $\underline{f}^{\prime \prime}, \underline{h}^{u}, \underline{g}^{u}, \underline{\hat{h}}^{\prime \prime}, \underline{\hat{g}}^{\prime \prime}$, to be evaluated for $t=\bar{t}$, are of class $C^{\infty}$ and we ask, locally around $(\bar{s}, \bar{x})$, where $\underline{\bar{x}}$ is a g.c point for $\underline{P}_{\mathscr{S}, \mathscr{F}}^{\prime \prime}(s, \bar{t})$ (or $\underline{P}_{\mathscr{F}}^{\mathscr{U}} u(s, \bar{t})$ if the Reduction-Ansatz could be used), for a smooth coordinate transformation $\underline{\varphi}_{i}$ of the following type

CCT1. $\underline{\varphi}_{\bar{t}}(s, \underline{x}):=\left(\underline{\varphi}_{\bar{t}}^{1}(s), \underline{\varphi}_{\bar{\tau}}^{2}(s, \underline{x})\right) \in \mathbb{R} \times \mathbb{R}^{n}$

CCT2. $\underline{\dot{\varphi}}_{\bar{t}^{\prime}}(s)>0$

CCT3. $\underline{\underline{\varphi}}_{\vec{t}}(\bar{s}, \underline{\bar{x}})=(0,0)\left(:=\left(\underline{0_{1}}, \underline{0_{n}}\right)\right)$.

This coordinate transformation preserves the special role of the parameter $s$. Moreover, it is canonical for each fixed value $s^{1}$ of $s$. We call $\underline{\varphi}_{\bar{\tau}}$ a canonical coordinate transformation (see [36]).

Type 1. Let $\bar{x}=0$ (zero-function) be a generalized critical trajectory of $\mathscr{P}_{\mathscr{S} \mathscr{I}}^{\prime \prime}(0)$ $((\bar{s}, \bar{x})=(0,0))$. Then in some neighbourhood $\mathscr{U}_{(\bar{s} . \bar{x})}$ of $(\bar{s}, \bar{x})$, with respect to the product-topology of both the natural topology and $C_{s}^{4}$, we have the following normal form:

$$
\begin{aligned}
\mathscr{I}^{u}(s, x) & :=\int_{a}^{b}\left(-2 \sum_{i=1}^{p_{f}} \pm x_{i} \cdot \dot{x}_{i}-\sum_{j=p_{f}+1}^{p_{f}+p_{u}} \pm \dot{x}_{j}+\eta(s, t)\right) d t \\
\dot{x} & =\frac{1}{t+a-2 b}\left(\begin{array}{cc}
I_{p_{f}+p_{a}} & \mathscr{O} \\
C(t) & D(t)
\end{array}\right) x+\left(\begin{array}{c}
\mathscr{O} \\
E(t)
\end{array}\right) u(s, t), \quad(c f . \text { also Note } 2), \\
x_{i}(a) & =0 \quad\left(i=p_{f}+p_{a}+1, \ldots, n\right) \\
x_{j}(a) & \geq 0 \quad\left(j=p_{f}+1, \ldots, p_{f}+p_{a}\right) .
\end{aligned}
$$

Here $I_{p_{f}+p_{a}}, \mathscr{O}$ may denote the $\left(p_{f}+p_{a}\right) \times\left(p_{f}+p_{a}\right)$-unit matrix and different zeromatrices of suitable sizes, respectively. The integers $p_{f}, p_{a}$ stand for the number of free or bound (active) variables of $\underline{x}$, that is, for the number of free or active inequality constraints which are free or active at $\underline{\bar{x}}$. We remember $p_{a}=\kappa=\kappa_{1}+\kappa_{2}, p_{a}+p_{f} \leq n$. These constraints and the equality constraints, too, have become linearized under $\underline{\varphi}_{\vec{r}}$. 
We note the next relations

$$
\begin{gathered}
x_{j}(t)=-\frac{t+a-2 b}{b-a} \frac{x_{j}(a)}{2}, \text { that is, } x_{j}(b)=\frac{x_{j}(a)}{2} \quad\left(j=1, \ldots, p_{f}+p_{a}\right), \\
\underline{f}^{\prime \prime}(s, a, \underline{x})=\frac{1}{2} \mathscr{N} \mathscr{F}(s, \underline{x}) \text { with } \mathscr{N} \mathscr{F}(s, \underline{x})=\sum_{i=1}^{p_{f}} \pm x_{i}^{2}+\sum_{j=p_{f}+1}^{p_{f}+p_{u}} \pm x_{j}+\rho(s),
\end{gathered}
$$

that is, $\begin{cases}=\sum_{i=1}^{p_{f}} \pm x_{i}^{2}+\sum_{j=p_{f}+1}^{p_{f}+p_{a}} x_{j}+\rho(s), & \text { if } x \text { is a Kuhn-Tucker trajectory } \\ =\sum_{i=1}^{p_{f}} x_{i}^{2}+\sum_{j=p_{f}+1}^{p_{f}+p_{u}} x_{j}+\rho(s), & \text { if } x \text { is a local minimum trajectory, }\end{cases}$

$$
\rho(s)=2 \int_{a}^{b} \eta(s, t) d t \quad(\eta \text { : depending on } u) \text {. }
$$

In particular, we see from (6) that the characteristic corresponding with the specific \pm distribution (local minimum, saddlepoint, local maximum) of a nondegenerate critical trajectory remains constant for small perturbations of the parameter. For a motivation and a proof of (6) (parametric Morse Lemma) by means of a suitable canonical coordinate transformation we refer to [36] (see also [55] and, for example, [6]). In that survey article, [36], a singularity theoretical approach to parametric optimization is also given.

We note that the part of Figure 1 which is due to type 1 is essentially based on the implicit function theorem.

Type 2. Let $\bar{x}=0$ be a corresponding generalized critical trajectory of $\mathscr{P}_{\mathscr{S} \mathscr{S}}^{\prime \prime}(0)$ $((\bar{s}, \underline{x})=(0,0))$. Here, maybe after a suitable transformation, we have the following normal form in a neighbourhood $\mathscr{U}_{(0,0)}$ of $(\bar{s}, \bar{x})$ :

$$
\begin{aligned}
\mathscr{I}^{u}(s, x) & :=\int_{a}^{b}\left(-2 \sum_{i=1}^{p_{f}} \pm x_{i} \cdot \dot{x}_{i} \pm 2\left(x_{p_{f}+1} \pm s\right) \dot{x}_{p_{f}+1}-\sum_{j=p_{f}+2}^{p_{f}+p_{a}} \pm \dot{x}_{j}+\eta(s, t)\right) d t \\
\dot{x} & =\frac{1}{t+a-2 b}\left(\begin{array}{cc}
I_{p_{f}+p_{u}} & \mathscr{O} \\
C(t) & D(t)
\end{array}\right) x+\left(\begin{array}{c}
\mathscr{O} \\
E(t)
\end{array}\right) u(s, t) \quad(\text { cf. Note } 2), \\
x_{i}(a) & =0 \quad\left(i=p_{f}+p_{a}+1, \ldots, n\right), \\
x_{j}(a) & \geq 0 \quad\left(j=p_{f}+1, \ldots, p_{f}+p_{a}\right) .
\end{aligned}
$$

Then, we also have

$$
\begin{aligned}
& \underline{f}^{\prime \prime}(s, a, \underline{x})=\frac{1}{2} \mathscr{N} \mathscr{F}(s, \underline{x}), \text { where } \\
& \mathscr{N} \mathscr{F}(s, \underline{x})=\sum_{i=1}^{p_{f}} \pm x_{i}^{2} \mp\left(x_{p_{f}+1}+s\right)^{2}+\sum_{j=p_{f}+2}^{p_{f}+p_{a}} \pm x_{j}+\rho(s)
\end{aligned}
$$

for example, $\eta=0$, hence, $\rho=0$. 
In the case of a local minimum of type 2 such a normal form has been suggested by Alina Ruiz (see [38]). It leads to the following objective functional

$$
\mathscr{I}^{u}(s, x):=\int_{a}^{b}\left(-2 \sum_{i=1}^{p_{f}} x_{i} \cdot \dot{x}_{i}+2\left(x_{p_{f}+1}+s\right) \dot{x}_{p_{f}+1}-\sum_{j=p_{f}+2}^{p_{f}+p_{a}} \dot{x}_{j}+\eta(s, t)\right) d t .
$$

Let us give a description of a characteristic problems' behaviour around $(\bar{s}, \underline{\bar{x}})$. As $s$ increases, on the one hand a local minimum proceeds from the (maybe relative) interior, hits the (maybe relative) boundary, and remains on it (see Figure 2). Such a branch of the critical set can be reflected as being due to that problem where the inequality constraint with vanishing Lagrange multiplier is deleted ( $c f .[30,38])$.

On the other hand, with increasing values of $s$ a local minimum on the boundary, being also due to treating the "critical" inequality with vanishing Lagrange multiplier as an equality constraint, disappears when it arrives at the point $(\bar{s}, \bar{x})=(0,0)$ (see Figure 3). For the fixed time $\bar{t}$ we start minimizing $\underline{f}^{u}(\cdot, \bar{t}, \cdot)$ over $\underline{M}_{0, \bar{t}}^{u}$ at $\underline{x}$ by means of a descent procedure. If $M_{0, t}^{u}$ is compact this guarantees that we reach another branch of $\underline{\Sigma}_{g c}(\bar{t})$, since the disappearing local minimum cannot be a global one ( $c f$. Figure 4 ). The descent at $\underline{\underline{x}}$ is possible since a cone of directions of descent is available.
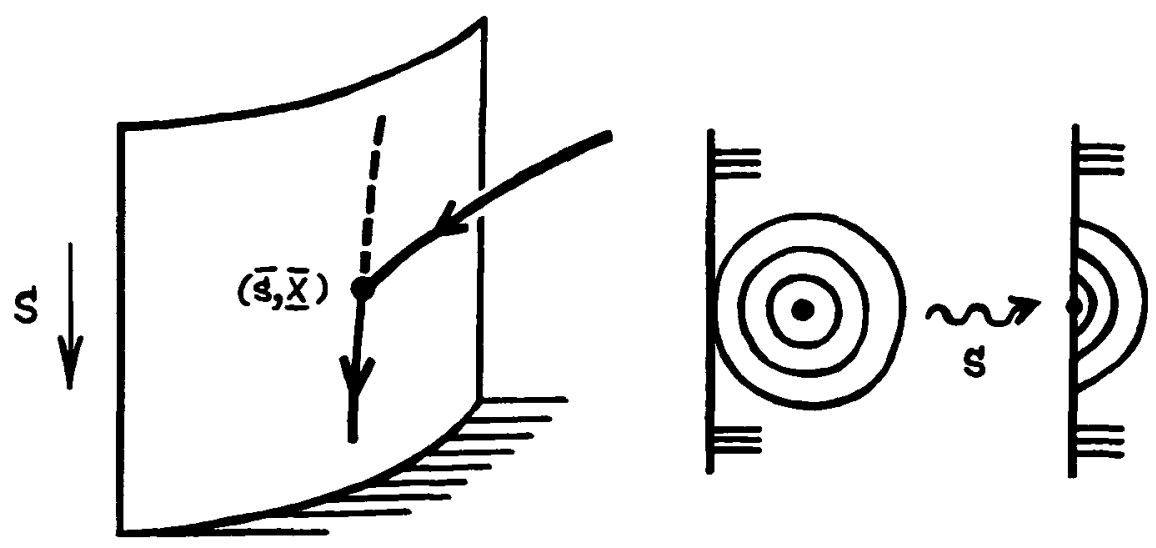

FIGURE 2. $\bar{x}$ of type 2: wandering local minimum (due to type 1 else) coming from the (relative) interior and entering the (relative) boundary at $(\bar{s}, \underline{\bar{x}})$.

Type 3. From singularity theory ([8], $c f$. also [36, 41, 68]) we learn that in suitable canonical coordinates here we have for the parametrized optimization problem the following normal form, locally in a neighbourhood $\mathscr{U}_{(0.0)}$ of $(\bar{s}, \bar{x})=(0,0)$ :

$$
\mathscr{I}^{u}(s, x):=\int_{a}^{b}\left(-\left(3 x_{1}^{2} \pm s\right) \dot{x}_{1}-\sum_{i=2}^{p_{f}} \pm 2 x_{i} \cdot \dot{x}_{i}-\sum_{j=p_{f}+1}^{p_{f}+p_{a}} \pm \dot{x}_{j}+\eta(s, t)\right) d t,
$$




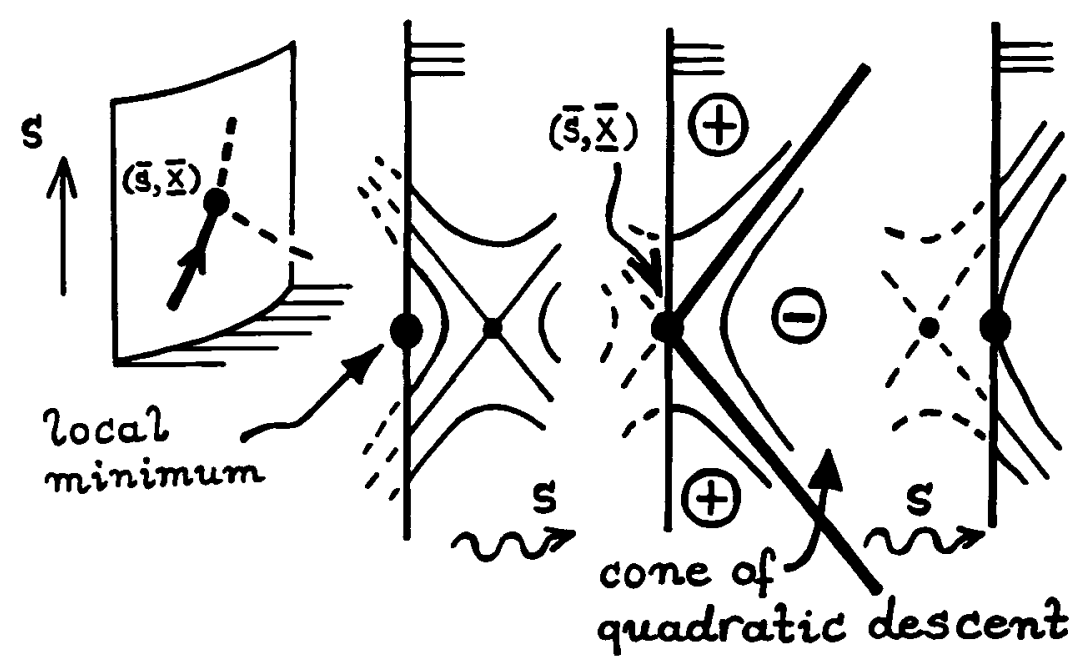

FIGURE 3. $\bar{x}$ of type 2: local minimum (due to type 1 else) wandering on the (relative) boundary and disappearing at $(\bar{s}, \underline{\bar{x}})$.

$$
\begin{aligned}
\dot{x} & =\frac{1}{t+a-2 b}\left(\begin{array}{cc}
I_{p_{f}+p_{a}} & \mathscr{O} \\
C(t) & D(t)
\end{array}\right) x+\left(\begin{array}{c}
\mathscr{O} \\
E(t)
\end{array}\right) u(s, t) \quad \text { (cf. Note 2), } \\
x_{i}(a) & =0 \quad\left(i=p_{f}+p_{a}+1, \ldots, n\right), \\
x_{j}(a) & \geq 0 \quad\left(j=p_{f}+1, \ldots, p_{f}+p_{a}\right) .
\end{aligned}
$$

We state the normal form of the objective function being a universal unfolding with normalized parameters (up to the factor $\frac{1}{2}$ ):

$$
\begin{aligned}
& \underline{f}^{\prime \prime}(s, a, \underline{x})=\frac{1}{2} \mathscr{N} F(s, \underline{x}), \text { where } \\
& \mathscr{N} \mathscr{F}(s, \underline{x})=x_{1}^{3} \pm s x_{1}+\sum_{i=2}^{p_{f}} \pm x_{i}^{2}+\sum_{j=p_{f}+1}^{p_{f}+p_{a}} \pm x_{j}+\rho(s) .
\end{aligned}
$$

This normal form which is correspondingly illustrated in Figure 5, gives rise to call $\bar{x}$ a quadratic turning trajectory or a fold trajectory (codimension 1). When passing the point $(\bar{s}, \bar{x})$ (or the curve $t \mapsto\left(\bar{s}, \bar{x}^{\bar{s}}(t)\right)$ along a path of local minima (or local minimum trajectories, respectively), the local minimum $\bar{x}_{\min }^{s}\left(=\bar{x}^{s}(\bar{t})\right)$ switches into a nondegenerate Kuhn-Tucker point $\bar{x}_{1}^{s}$ of quadratic index one. That is, by definition, at such a point precisely one eigenvalue of the restricted Hessian of the Lagrange function is negative. For $p_{f}>1$ such a point is a saddlepoint. Hence the path of 


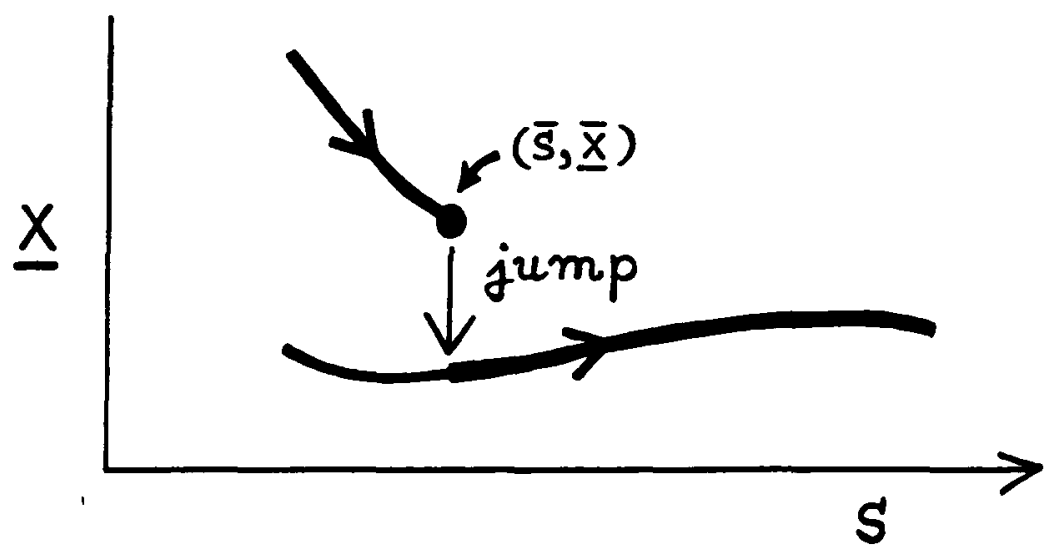

FIGURE 4. $\bar{x}$ of type 2: jump at $(\bar{s}, \underline{x})$ from one branch of local minima to another branch of local minima.

local minima stops at $(\bar{s}, \underline{\bar{x}})$, and for $s$ near $\bar{s}$ the local minimum $\underline{\underline{x}}_{\min }^{s}$ for $\left.\underline{f}^{\prime \prime}(s, \bar{t}, \cdot)\right|_{\underline{M}_{s, i}^{\prime \prime}}$ cannot be a global one. Now, as we have

$$
\underline{\underline{v}}^{s}:=\frac{\bar{x}_{1}^{s}-\overline{\underline{x}}_{\min }^{s}}{\left\|\underline{\bar{x}}_{1}^{s}-\underline{\bar{x}}_{\min }^{s}\right\|} \longrightarrow \underline{\bar{v}} \quad(s \longrightarrow \bar{s}),
$$

with $\underline{\underline{v}}$ being a direction of cubic descent, there is the possibility to jump to another branch of local minima. This can be done in the case of compactness of the feasible set $\underline{M}_{0, \bar{i}}^{u}$ by means of a descent procedure for $s=\bar{s}$, starting at the point $(\bar{s}, \bar{x})$ (see Figure 5; $c f .[38])$.

Type 4. By means of a canonical coordinate transformation this case could essentially be reduced to the case of one (in)equality constraint. In this sense we have as a first easy example, in dimension $n=2$ with one equality constraint, the following normal form. It actually fulfills the transversality conditions which characterize type 4 (cf. [38]). Namely,

$$
\begin{aligned}
\mathscr{I}^{\prime \prime}(s, x) & :=\int_{a}^{b}\left(\mp 4 \dot{x}_{1} \mp \dot{x}_{2}+\eta(s, t)\right) d t, \\
\dot{x} & \left.=\frac{1}{t+a-2 b} x \quad \text { (remember Note } 2\right), \\
x_{1}^{2}(a)-x_{2}^{2}(a) & =-s .
\end{aligned}
$$

For the case of general $n \geq 2$, for a local minimum trajectory $\bar{x}=0$ at the parameter $\bar{s}=0$, and of one inequality constraint, we state the two possible cases 4 .I and 4.II. We note that the structure of problems in dimension $n$ is already implied in problems of 


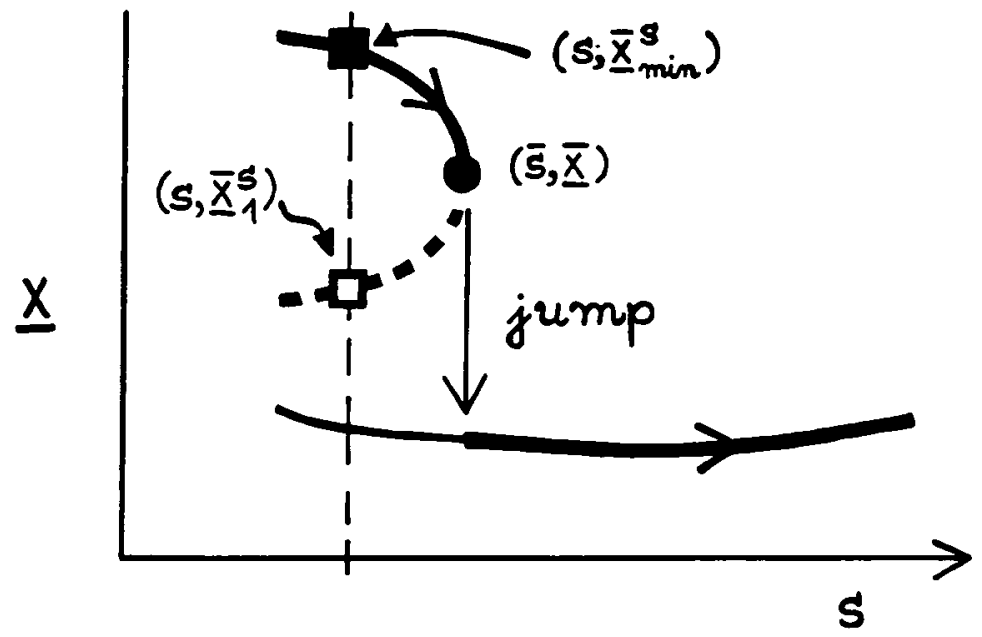

FIGURE 5. $\bar{x}$ of type 3 : jump at $(\bar{s}, \underline{x})$ from one branch of local minima to another branch of local minima.

low dimensions. This possibility of reducing the dimension is known in the research of structural stability (cf. $[37,64])$. Namely, we have

$$
\begin{aligned}
\mathscr{I}^{\prime \prime}(s, x) & :=\int_{a}^{b}\left(-4 \dot{x}_{1}-\sum_{j=2}^{n} \dot{x}_{j}+\eta(s, t)\right) d t, \\
\dot{x} & \left.=\frac{1}{t+a-2 b} x \quad \text { (remember Note } 2\right)
\end{aligned}
$$

and precisely one of the two conditions

$$
\begin{aligned}
& \text { 4.I. } \quad x_{1}^{2}(a)-\sum_{j=2}^{n} x_{j}^{2}(a) \geq-s, \\
& \text { 4.II. } \quad-\sum_{j=1}^{n} x_{j}^{2}(a) \geq s .
\end{aligned}
$$

Case 4.I. Here we are in the situation as illustrated in Figure 6.

Provided that $\underline{M}_{0, i}^{\prime \prime}$ is compact, starting for $s<0$ close to 0 , at the point $\bar{x}_{\max }^{s}$ (= $\left.\bar{x}_{\max }^{s}(\bar{t})\right)$ with a descent procedure we shall end up in a local minimum which is different from $\underline{\underline{x}}_{\min }^{s}$. So, by means of a jump we have arrived at another branch of local minima.

Case 4.II. Figure 7 shows us that in this case a jump to another branch of local minima cannot be expected (see also [38]). 


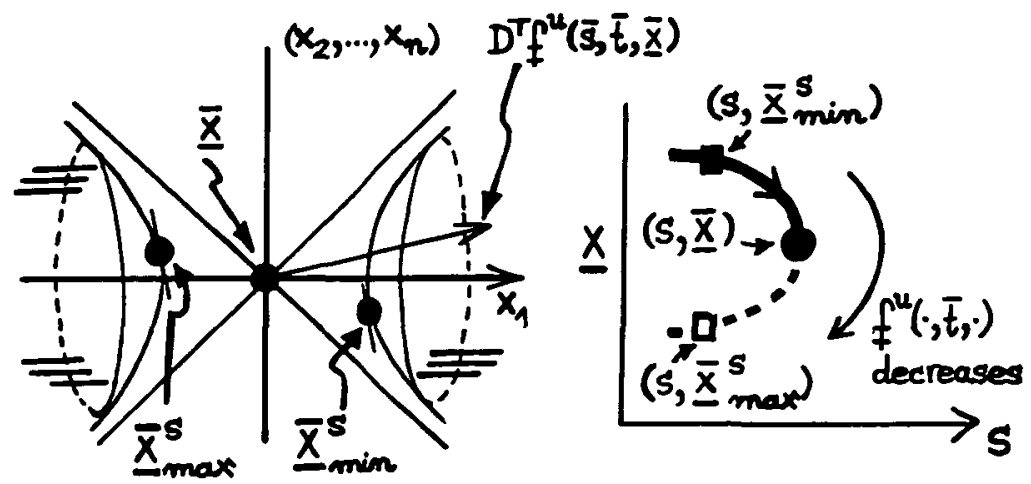

FigURE 6. $\bar{x}$ of type 4 : case $4 . I$.

Type 5. By means of a careful study of the detailed introduction of type 5 in [30], with a suitable choice of the multipliers in the following characteristic equations (with renumbered inequalities):

$$
\begin{aligned}
& \sum_{i \in \underline{L}} \lambda_{i} D_{\underline{x}} \underline{h}_{i}^{u \prime}(\bar{s}, \bar{t}, \underline{\bar{x}})+\sum_{j=1}^{p_{u}} \mu_{j} D_{\underline{x}} \underline{g}_{j}^{\mathscr{U}, u}(\bar{s}, \bar{t}, \underline{\bar{x}})=0 \quad \text { (not all } \lambda_{i}, \mu_{j} \text { vanishing), } \\
& \sum_{i \in \underline{I}} \alpha_{i} D_{(s . \underline{w})} \underline{h}_{i}^{u}(\bar{s}, \bar{t}, \underline{\bar{x}})+\sum_{j=1}^{p_{a}} \beta_{j} D_{(s . \underline{w})} \underline{g}_{j}^{\mathscr{U} u}(\bar{s}, \bar{t}, \underline{\bar{x}})=D_{(s, \underline{x})} \underline{f}^{u}(\bar{s}, \bar{t}, \bar{x})
\end{aligned}
$$

by $\lambda_{i}=\mu_{j}=1, \alpha_{i}=0, \beta_{j}= \pm j\left(i \in \underline{I}, j \in\left\{1, \ldots, p_{a}\right\}\right)$, we get the following easy example. Namely, the following model, defined in a neighbourhood $\mathscr{U}_{(\bar{s}, \bar{x})}$ of $(\bar{s}, \bar{x})=(0,0)$, is suitable for a generalized critical trajectory $\bar{x}$ being of type 5 for $\mathscr{P O P}_{\mathscr{S P}}^{\prime \prime}(\bar{s})$ :

$$
\begin{aligned}
\mathscr{I}^{\prime \prime}(s, x) & :=\int_{a}^{b}\left(-\sum_{j=1}^{p_{a}} \pm j \dot{x}_{j}+\eta(s, t)\right) d t \\
\dot{x} & =\frac{1}{t+a-2 b}\left(\begin{array}{cc}
I_{p_{f}+p_{a}} & \mathscr{O} \\
C(t) & D(t)
\end{array}\right) x+\left(\begin{array}{c}
\mathscr{O} \\
E(t)
\end{array}\right) u(s, t) \quad \text { (cf. Note 2), } \\
x_{i}(a) & =0 \quad\left(i=p_{a}+1, \ldots, n\right), \\
-\sum_{j=1}^{n} x_{j}(a) & =s \\
x_{j}(a) & \geq 0 \quad\left(j=1, \ldots, p_{a}\right) .
\end{aligned}
$$

For a generalized critical point $\underline{x}$, of type 5 and due to the parameter value $s=\bar{s}$, we know in general that $\underline{\Sigma}_{g c}(\bar{t})$ locally around $(\bar{s}, \underline{\bar{x}})$ consists of exactly $p_{a}$ (half) curves, 

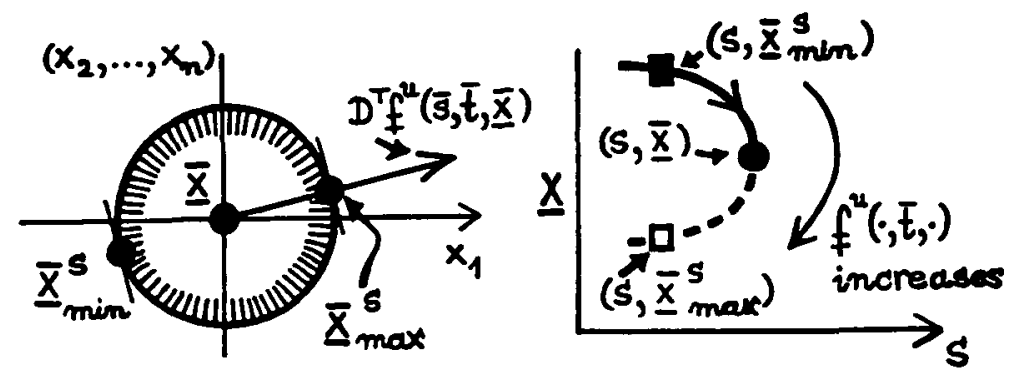

FIGURE 7. $\bar{x}$ of type 4 : case $4 . I I$.

each of them emanating at $(\bar{s}, \underline{\bar{x}})$ or ending at $(\bar{s}, \underline{\bar{x}})$ for increasing $s$ (see $[30,38])$.

Case 5.I. The Mangasarian-Fromovitz constraint qualification is satisfied at $\underline{\bar{x}}$.

Then precisely one of the emanating (half) curves consists of local minima. Hence we can proceed with tracing local minima (pathfollowing).

Case 5.II. The Mangasarian-Fromovitz constraint qualification is violated at $\bar{x}$.

Then the actual component of the feasible set becomes empty. Whenever a branch of local minima stops at $(\bar{s}, \underline{x})$, a simple jump to another branch of local minima cannot be performed.

The cases 5.I and 5.II are illustrated in Figure 8.

Types 6-8. Here, the degeneracy is always caused at a problem of the lower stage. Hence, easy examples can be given, at first referring there to problems from finite optimization which are in "normal form". Then we refer to problems of the upper stage which transversally fit to these. However, let us only give an example of a generalized critical trajectory $\bar{x}=0$ of type 6 at $\bar{s}=0$. Here we evaluate the corresponding example in semi-infinite optimization from [20] $(n=2)$. In a neighbourhood $\mathscr{U}_{(0.0)}$ of $(\bar{s}, \bar{x})$ we have

$$
\begin{aligned}
\mathscr{I}^{\prime \prime}(s, x) & :=\int_{a}^{b}\left(2 x_{1} \dot{x}_{1}+2\left(x_{2}+1\right) \dot{x}_{2}+\eta(s, t)\right) d t, \\
\dot{x} & \left.=\frac{1}{t+a-2 b} x \quad \text { (cf. Note } 2\right), \\
-x_{1}^{2}(a)-x_{2}(a)+\left(y-x_{1}(a)+s\right)^{2} & \geq 0 \quad \text { for all } y \in \mathbb{R} \text { with }(y-s) \cdot(1-y) \geq 0 .
\end{aligned}
$$




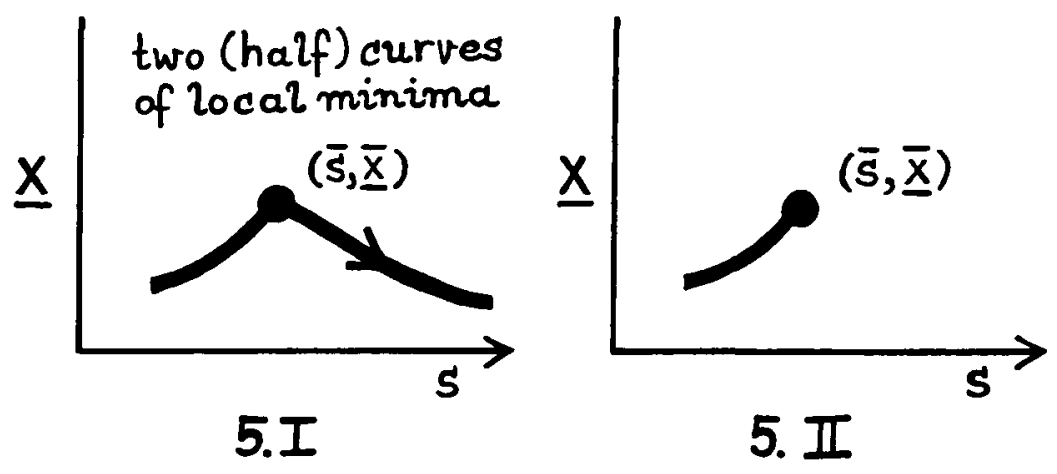

FIGURE $8 . \bar{x}$ of type 5: case 5.1 and case 5.II.

Here, our inequality constraint on $x$ may by means of the flow be induced from an inequality constraint of boundary value form.

The description of $\underline{M}_{0 . \bar{t}}^{u}$ and $\underline{\Sigma}_{g c}(\bar{t})$ near $\underline{\bar{x}}=0$ given in Figure 9 can be realized by deleting the inequality constraint of the lower stage (see $\bar{x}_{d}^{s}$ ), or by treating this constraint as an equality (see $\bar{x}_{e}^{s} ; c f$. also type 2 and see [20]), respectively.

We note that in this example the branch of local minima, say of local minimum trajectories, consists of a single element.

Detailed information on the types 7, 8 is given in [35].

For all stated problems in normal form we remark that one can turn from the state constraints at time $t=a$ to any time $t \in[a, b]$ by means of the flow. Here for example, in the case of our special differential equations, we substitute $x_{j}(a)=$ $-2(b-a) x_{j}(t) /(t+a-2 b)$, for all the coordinates $j$ which are involved in the integrand.

\section{Concluding remark}

In Section 1 we also indicated the concept of structural stability of our optimal control problem. Whenever the latter problem is equivalent to a finite optimization problem with compact feasible set, maybe after applying the Reduction-Ansatz, we can characterize the structural stability essentially by means of the (extended) Mangasarian-Fromovitz constraint qualification and of strong stability, meaning the continuous dependence of the Kuhn-Tucker points on the functional data. This fact underlines the importance of the extended Mangasarian-Fromovitz constraint qualification condition which also plays a part in our classification by means of eight generic 


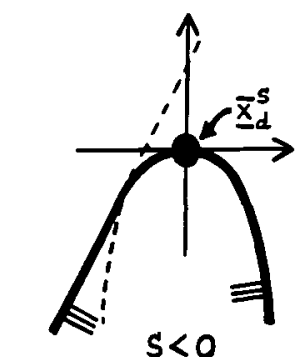

$s<0$

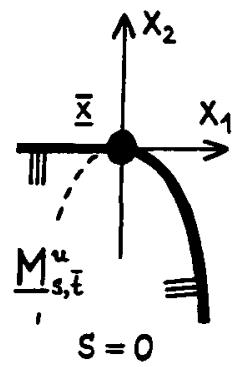

$S=0$

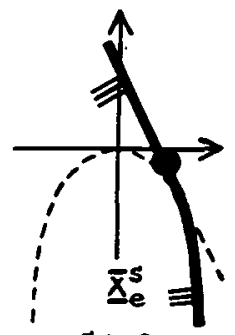

$s>0$

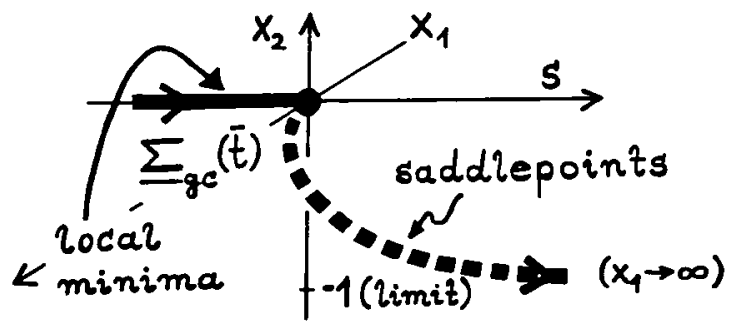

FIGURE 9. $\bar{x}$ of type 6: an example; $\underline{M}_{s, \bar{\tau}}^{\prime \prime}$ and a component of $\underline{\Sigma}_{g c}(\bar{t})$.

types. In particular we see that the extremal set of the original problem (or at the lower stage, respectively) may become empty at a generalized critical trajectory of type 5 (or 8 , respectively). From Figure 1 we also conclude that generalized critical points and trajectories of types 2-5 do not need to be strongly stable. However, at a strongly stable generalized critical point or trajectory, more than one Lagrange multiplier $\mu_{j}$ or more than one eigenvalue $\sigma_{\ell}$ of the Hessian matrix of the Lagrangian on the tangent space may vanish. We note that strong stability is a weaker condition than nondegeneracy.

The condition of structural stability is numerically reasonable, for example, descent is preserved under perturbation. But the study of structural stability does moreover give geometrical insights into the problems' behaviour ( $c f .[37,64])$. Its characterization can be extended to max-type functions, but it becomes more difficult in the case of semi-infinite models and in the case of noncompact feasible sets. The same can be stated for our generic classification.

In [66] and in this article we had to look at subsets of the entire space of the problems' defining functions, for example, by means of an integrability assumption. From physicists who are concerned with structural stability we learn that such restrictions of the whole space can be very natural and practical. For an interesting exposition of this fact in the context of structural formation and renormalization techniques we refer to $[9,10]$. In our context, maybe nonstandard analysis [24] will in future turn out to give helpful explanations [63]. From the viewpoint of both optimization and structural formation, the articles $[13,14,16]$ give more information and motivation. 
Some directions of further research have already been pointed out in the last sections. Additionally, we recognize the interest in a comparison of this research with the classical conditions and numerical (for example, "shooting") methods in optimal control theory as studied in, for example, [4, 5, 42, 46, 48, 50, 51, 52, 53, 54, 56, 71]. Moreover, besides our approach with generalized critical points and their branches, the Newton flows stand for another concept from optimization (see [11, 12, 28, 31]; $c f$. also $[3,15,26]$ for related research). These flows may more and more become a helpful tool ([45]), also in optimal control theory.

We conclude referring to further different investigations on Newton's method by Smale, Yomdin and others, for example, [58, 59, 60,70]; and with the following reflection on the relation between optimal control theory and optimization.

In this article we traced back an optimal control problem to an optimization problem. The reverse direction could also be very interesting, namely to decouple the constraints of an optimiztion problem close to a maybe degenerate (generalized) critical point, by means of distributing these constraints together with the objective function to different times $t$ with the help of a suitable flow. This could lead to a "desingularized" family of optimization problems which constitute an optimal control problem, or a discretization of such a problem, to be studied.

\section{Acknowledgement}

The author thanks Professors B. D. Craven, W. Krabs and H.Th. Jongen for their valuable advice and encouragement.

\section{References}

[1] E. Allgower and K. Georg, Introduction to Numerical Continuation Methods (Springer, 1990).

[2] H. Amann, Gewöhnliche Differentialgleichungen (Walter de Gruyter, 1979).

[3] A. S. Antipin, "Proximal differential systems with feedback control", Russian Acad. Sci. Dokl. Math. 47 (1993) 183-186.

[4] A. V. Arutyunov and N. T. Tynyanski1, "On necessary conditions for a local minimum in optimal control theory", Soviet Math. Dokl. 29 (1984) 176-179.

[5] A. V. Arutyunov, D. B. Silin and L. G. Zerkalov, "Maximum principle and second-order conditions for minimax problems of optimal control", J. Optimization Theory and Applications 75 (1992) 521-533.

[6] E. R. Avakov, A. A. Agrachev and A. V. Arutyunov, "The level set of a smooth mapping in a neighbourhood of a singular point, and zeros of a quadratic mapping", Math. USSR Sbornik 73 (1992) 455-466.

[7] B. Bank, J. Guddat, D. Klatte, B. Kummer and K. Tammer, Non-Linear Parametric Optimization (Akademie-Verlag, Berlin, 1982). 
[8] Th. Bröcker and L. Lander, Differentiable Germs and Catastrophes, London Math. Society Lecture Note Series 17 (Cambridge University Press, 1975).

[9] L.-Y. Chen, N. Goldenfeld, Y. Oono and G. Paquette, "Selection, stability and renormalization", Physica A 204 (1994) 111-133.

[10] L.-Y. Chen, N. Goldenfeld and Y. Oono, "Renormalization group theory for global asymptotic analysis", preprint, University of Illinois at Urbana-Champaign (1994).

[11] I. Diener, "Trajectory nets connecting all critical points of a smooth function", Mathematical Programming (1986) 340-352.

[12] I. Diener, Globale Aspekte des kontinuierlichen Newton-Velfahrens (Habilitationsschrift, University of Göttingen, Germany, 1994).

[13] A. W. M. Dress, "Die Formensprache der Natur als Gegenstand der Mathematik", Forschung an der Universität Bielefeld 5. University of Bielefeld (1992) .

[14] A. W. M. Dress, D. Huson and A. Müller, "Symmetrie und Topologie von Riesenmolekülen, supramolekularen Clustern und Kristallen", preprint. University of Bielefeld (1993).

[15] L. C. Evans and J. Spruck, "Motion of level sets by mean curvature I.", J. Differential Geometry 33 ( 1991 ) 635-681.

[16] H. Frauenfelder and P. G. Wolynes, "Biomolecules: where the physics of complexity and simplicity meet", Physics Today (1994) 58-64.

[17] J. Guddat, H.Th. Jongen and J. Rückmann, "On stability and stationary points in nonlinear optimization", J. Austral. Math. Soc. Ser: B 28 (1986) 36-56.

[18] J. Guddat and H.Th. Jongen, "Structural stability in nonlinear optimization", Optimization 18 (1987) 617-631.

[19] F. Guerra Vasquez, J. Guddat and H.Th. Jongen, Parametric Optimization: Singularities. Pathfollowing and Jumps (John Wiley, 1990).

[20] R. Hettich, H.Th. Jongen and O. Stein, "On continuous deformations of semi-infinite optimization problems", in Approximation and Optimization in the Caribbean II (eds. M. Florenzano, J. Guddat, M. Jimenez, H.Th. Jongen, G. Lopez Lagomasino and F. Marcellan), (Peter Lang Verlag, Frankfurt a.M., 1995), 406-424.

[21] R. Hettich and P. Zencke, Numerische Methoden der Approximation und semi-infiniten Optimiertung (Teubner Studienbücher, Stuttgart, 1982).

[22] M. W. Hirsch, Differential Topology (Springer Verlag, 1976).

[23] B. R. Hunt, T. Sauer and J. A. Yorke, "Prevalence: a translation-invariant "almost every" on infinite dimensional spaces", Bulletin of the American Mathematical Society 27 (1992) 217-238.

[24] A. E. Hurd and P. A. Loeb, An Introduction to Nonstandard Real Analysis (Academic Press, Inc., 1985).

[25] N. H. Ibragimov, "Sophus Lie and harmony in mathematical physics, on the 150th anniversary of his birth", The Mathematical Intelligencer 16 (1994) 20-28.

[26] T. Ilmanen, "The level-set flow on a manifold", Proc. Symposia in Pure Marhemarics 54, part I (1993) 193-203.

[27] H.Th. Jongen, P. Jonker and F. Twilt, Nonlinear Optimization in $\mathbb{R}^{\prime \prime}, I$. Morse Theory, Chebychev Approximation (Peter Lang Verlag, Frankfurt a.M., Bern, New York, 1983).

[28] H.Th. Jongen, P. Jonker and F. Twilt, Nonlinear Optimization in $\mathbb{R}^{\prime \prime}$, II. Transversality, Flou's. Parametric Aspects (Peter Lang Verlag, Frankfurt a.M., Bern, New York, 1986).

[29] H.Th. Jongen, P. Jonker and F. Twilt, "One-parameter families of optimization problems: equality constraints", J. Optimization Theory and Applications 48 (1986) 141-161.

[30] H.Th. Jongen, P. Jonker and F. Twilt, "Critical sets in parametric optimization", Mathematical Programming 34 (1986) 333-353.

[31] H.Th. Jongen, P. Jonker and F. Twilt, "A note on Branin's method for finding the critical point of smooth function", in Parametric Optimization and Related Topics (ed. J. Guddat et al.), (Akademie- 
Verlag, Berlin, 1987) 209-228.

[32] H.Th. Jongen and D. Pallaschke, "On linearization and continuous selections of functions", $O p$ timization 19 (1988) 343-353.

[33] H.Th. Jongen, F. Twilt and G.-W. Weber, "Semi-infinite optimization: structure and stability of the feasible set", J. Optimization Theory and Applications 72 (1992) 529-552.

[34] H.Th. Jongen, J.-J. Rückmann and G.-W. Weber, "One-parametric nonlinear optimization: on the stability of the feasible set", SIAM J. Optimization 4 (1994) 637-648.

[35] H.Th. Jongen and O. Stein, "On generic one-parametric semi-infinite optimization", preprint, University of Trier, Trier; Germany (1995).

[36] H.Th. Jongen and G.-W. Weber, "On parametric nonlinear programming", Annals of Operations Research 27 (1990) 253-284.

[37] H.Th. Jongen and G.-W. Weber, "Nonlinear optimization: characterization of structural stability", J. Global Optimization 1 (1991) 47-64.

[38] H.Th. Jongen and G.-W. Weber, "Nonconvex optimization and its structural frontiers", in Modern Methods of Optimization (eds. W. Krabs and J. Zowe), Lecture Notes in Econom. and Math. Systems 378 (1992) 151-203.

[39] H.Th. Jongen and G.-W. Weber, personal communication, 1994.

[40] H.Th. Jongen and G. Zwier, "On the local structure of the feasible set in semi-infinite optimization", in Parametric Optimization and Approximation (eds. B. Brosowski and F. Deutsch), ISNM 72, (Birkhäuser Verlag, Basel-Boston-Stuttgart, 1985) 185-202.

[41] H.Th. Jongen and G. Zwier, "On regular semi-infinite optimization", in Infinite Programming (eds. E. J. Anderson and A. B. Philpott), Lecture Notes in Econom. and Math. Systems 259, (Springer Verlag, 1985) 53-64.

[42] R. Klötzler, "Transportfluß-Optimierung", GMÖOR Newsletter 2 (1994) 3-10.

[43] M. Kojima, "Strongly stable stationary solutions in nonlinear programs", in Analysis and Computation of Fixed Points (ed. S. M. Robinson), (Academic Press, 1980) 93-138.

[44] M. Kojima and R. Hirabayashi, "Continuous deformations of nonlinear programs", Mathematical Programming Study 21 (1984) 150-198.

[45] W. Krabs and G.-W. Weber, personal communication, Darmstadt, Germany, 1994.

[46] F. Lempio and H. Maurer, "Differential stability in optimal control problems", Appl. Math. Optim. 5 (1979) 283-295.

[47] K. Malanowski, remark, Güstrow, Germany, 1991.

[48] K. Malanowski, "Regularity of solutions in stability analysis of optimization and optimal control problems", Control and Cybernetics 23 (1994) 61-86.

[49] O. L. Mangasarian and S. Fromovitz, "The Fritz John necessary optimality condition in the presence of equality and inequality constraints", J. Math. Anal. Appl. 17 (1967) 37-47.

[50] H. Maurer, "Numerical solution of singular control problems using multiple shooting", J. Optimization Theory and Applications 18 (1976) 235-257.

[51] H. Maurer, "On optimal control problems with bounded state variables and control appearing linearly", SIAM J. Control and Optimization 15 (1977) 345-362.

[52] H. Maurer, "Differential stability in infinite-dimensional nonlinear programming", Appl. Math. Optim. 6 (1980) 139-152.

[53] H. Maurer and H.-J. Pesch, "Solution differentiability for parametric nonlinear control problems with control-state constraints", Control and Cybernetics 23 (1994) 201-227.

[54] H. Maurer and S. Pickenhain, "Second-order sufficient conditions for control problems with mixed control-state constraints", preprim, University of Münster; Germany (1993).

[55] J. Milnor, "Morse theory", Annals of Mathematical Studies 51 (Princeton University Press, 1963).

[56] L. S. Pontryagin, V. G. Boltryanski, R. V. Gamkrelidze and E. F. Mishchenko, The Mathematical Theory of Optimal Processes (Interscience Publishers, J. Wiley, 1962). 
[57] Th. Rupp, "Kontinuitätsmethoden zur Lösung einparametrischer semi-infiniter Optimierungsprobleme", thesis, University of Trier, 1988.

[58] S. Smale, "Algorithms for solving equations", in Proc. Int. Congr. Math. I (ed. M. S. Gleason), (AMS, Berkeley, 1986) 172-195.

[59] S. Smale, "Complexity of Bezout's theorem I. Geometric aspects", Journal of the Americal Mathematical Society 6 (1993) 459-501.

[60] S. Smale, "Complexity of Bezout's theorem IV. Probability of success; extensions", SIAM J. Numerical Analysis, to appear.

[61] E. H. Spanier, Algebraic Topology (McGraw-Hill, 1966).

[62] R. Thom, Stabilité Structurelle et Morphogénèse: Essai d'une Théorie Générale des Modéles (W. A. Benjamin, Reading, MA, 1972).

[63] Th. Walter and G.-W. Weber, personal communication, Darmstadt, 1994.

[64] G.-W. Weber, Charakterisiertung struktureller Stabilität in der nichtlinearen Optimierung, thesis, Technical University of Aachen, Aachener Beiträge zur Mathematik 5, Augus-tinus-Buchhandlung, Aachen, Germany, 1992.

[65] G.-W. Weber, "Minimization of a max-type function: characterization of structural stability", in Parametric Optimization and Related Topics III (eds. J. Guddat, H.Th. Jongen, B. Kummer and F. Nožička), (Peter Lang Verlag, Frankfurt a.M., Bern, New York, 1993) 519-538.

[66] G.-W. Weber, "Optimal control theory: on the global structure and connections with optimization. Parts 1,2”, preprints, Darmstadt University of Technology, Darmstadt, Germany, 1995.

[67] W. W. E. Wetterling, "Definitheitsbedingungen für relative Extrema bei Optimierungs- und Approximationsaufgaben", Numer. Math. 15 (1970) 122-136.

[68] Y. Yomdin, "On the local structure of a generic central set", Compositio Mathematica (1981) 225-238.

[69] Y. Yomdin, "Metric semialgebraic geometry with applications in smooth analysis", preprint, Weizman Institute of Science, Rehovot, Israel (1988).

[70] Y. Yomdin, "Sard's theorem and its improved versions in numerical analysis", preprint, Weizman Institute of Science, Rehovot, Israel (1988).

[71] T. Zolezzi, "Well posedness criteria in optimization with application to the calculus of variations", Nonlinear Analysis - Theory, Methods, Applications, to appear. 